\title{
Polar decomposition based corotational framework for triangular shell elements with distributed loads
}

\author{
Federica Caselli and Paolo Bisegna*,† \\ Department of Civil Engineering and Computer Science, University of Rome 'Tor Vergata', 00133 Rome, Italy
}

\begin{abstract}
SUMMARY
A polar decomposition based corotational formulation for deriving geometrically nonlinear triangular shell elements is proposed. This formulation is novel in two aspects. (1) Original formulas for the projector operator and its variation are presented, leading to simple algorithms for the computation of the nodal residual vector and of the consistent tangent stiffness tensor. (2) For the first time in the context of a corotational kinematic description, a rigorous treatment of distributed dead and follower loads is performed, thoroughly accounting for the various contributions entailed in the residual vector and in the tangent stiffness. Numerical simulations of popular benchmark problems are reported, showing the effectiveness of the proposed approach. An accessible and adaptable MATLAB toolkit implementing the present formulation is provided as supplementary material.* Copyright (C) 2013 John Wiley \& Sons, Ltd.
\end{abstract}

Received 31 January 2013; Revised 19 April 2013; Accepted 24 April 2013

KEY WORDS: $\quad$ structures; FEM; shells; corotational formulation; consistent linearization; distributed loads

\section{INTRODUCTION}

Shell structures are widely used in engineering practice, and the development of efficient numerical methods for their analysis well into the nonlinear regime has been receiving continuous attention in last decades. Many applications in structural, aerospace, and biomedical engineering involve complex multiphysics problems and demand for accurate yet simple computational tools. Among the latter, the FEM has promoted the most significant advances in computational shell mechanics. Different types of finite elements have been developed for the analysis of shell structures, including 2D elements based on a shell theory, degenerated shell elements, and solid-shell elements. The most distinctive feature of the latter is that they do not possess any rotational DOFs [1]; hence, difficulties associated to the pseudo-vectorial nature of finite rotations are circumvented. On the other hand, the treatment of rotational DOFs in degenerated shell elements and 2D elements based on a shell theory may benefit from a corotational approach, which is characterized by the ability to handle easily geometric nonlinearities induced by finite rotations, decoupling them from element computations. In fact, the corotational approach represents an effective technique for deriving degenerated/2D shell elements ideally suited for applications characterized by complex features such as biological materials, contact, or fluid-structure interaction.

The corotational formulation is based on the idea of separating rigid body motions from strain producing ones. It was originally introduced by Wempner [2] and Belytschko and coworkers [3], and indeed presents similarities with the 'natural approach' of Argyris et al. [4]. Rankin and Brogan [5] introduced the concept of 'element-independent corotational formulation' and produced a technique

\footnotetext{
*Supporting information may be found in the online version of this article.

*Correspondence to: Paolo Bisegna, Department of Civil Engineering and Computer Science, University of Rome 'Tor Vergata', 00133 Rome, Italy.

†E-mail: bisegna@uniroma2.it 
whereby the corotational procedures were added to existing elements without, in any way, altering the latter. The element-independent corotational formulation is especially useful in problems involving large displacements and small strains. In fact, in those cases, existing high-performance linear elements can be reused as core elements in the geometrically nonlinear context, after large rigid body motions have been filtered out. The issue of consistent derivations of the nodal residual vector and of the tangent stiffness tensor was firstly addressed by Nour-Omid and Rankin $[6,7]$ and Crisfield $[8,9]$. A unified theoretical framework of small-strain corotational finite elements was presented by Felippa and Haugen [10]. Crisfield and Moita extended the corotational formulation to include finite strain [11,12]. That issue was also addressed by Rankin [13] and Areias et al. [14]. The development of shell elements based on a corotational framework has generated an increased amount of interest in last years: a curved triangular shell element employing assumed strains was proposed in [15], a rotation-free triangle was formulated in [16], and flat triangular shell elements for the analysis of thick shell or laminated composite structures were, respectively, formulated in [17] and [18], to name but a few examples. A review on geometrically nonlinear analysis of shell structures based on the corotational approach and flat triangular shell elements has been presented in [19].

The first issue in the development of a corotational procedure is the identification of the rigid body motion, also known as the shadowing problem [20]. Several proposals have been put forth in the literature; for triangular shell elements, they include side alignment [7], least square angular fit [10], and fit according to minimum least square deformation [21]. Some desirable properties have been listed in [10] as criteria to be followed in the identification of the rigid motion: (1) versatility (i.e., suitability to statics, dynamics, rigid bodies, and nonstructural bodies); (2) ability to return a bodyattached frame for rigid bodies; (3) invariance with respect to element node ordering; and (4) finite stretch patch test satisfaction (i.e., if a group of elements is subjected to a uniform stretch, then no spurious deformational rotations should appear). The latter property is not satisfied by the proposals cited earlier, because large strains induce finite rotations of directions not aligned to principal strain directions [14]. The key idea to satisfy the finite stretch patch test in 2D problems is due to Jeutter and Cescotto [22] and is based on the concept of null local spin at the center of the element. Crisfield extended the previous formulation to 3D continua and shell elements, proposing an approach based on the polar decomposition of the element motion [11, 12,23]. In particular, for three-node triangular shell elements, in [23], it is suggested to identify the rigid body motion by means of the polar decomposition of the gradient of the homogeneous transformation mapping the element nodes onto their current positions. This approach allows to satisfy all the desirable properties previously listed. A two-step strategy for the computation of the aforementioned polar decomposition has been proposed by Areias et al. [14]: The homogeneous transformation is regarded as the composition of a side-aligning in-plane transformation, to which a 2D polar decomposition applies, followed by a rigid transformation.

Once identified, the rigid body motion is purged out from the total displacements, thus yielding the filtered nodal parameters. The latter represents the input for the element routines that provide the nodal residual vector and the tangent stiffness tensor at the so-called core-element level. To lift the nodal residual vector to the overall level, the crucial ingredient is the so-called projector operator, able to extract the deformational part from incremental displacements. Analogously, the variation of the projector operator is required to lift the core-element tangent stiffness tensor to the overall level in a consistent way.

To the best of authors' knowledge, explicit formulas for the projector operator and its variation have not been reported in the literature for a polar decomposition based corotational framework for three-node triangular shell elements. A spin-fitter tensor forming the projector operator was introduced in [23], but no explicit expression was supplied, and its variation was neglected in the derivation of the tangent stiffness tensor. A consistent linearization, in the framework of the two-step strategy cited earlier, was achieved in [14], but resulting formulas, derived by means of symbolic software tools, were not reported. The first main contribution of the present work is the derivation of simple and elegant formulas for the computation of the projector operator and its variation, thus providing a simple algorithm for the computation of the nodal residual vector and of the consistent tangent stiffness tensor. These formulas are crucial for obtaining highly efficient implementations. 
In passing, it is noted that as opposite to most of the corotational literature, an intrinsic coordinatefree notation is used, which results in a particularly neat derivation.

The second main result of this work concerns the treatment of distributed loads, which play a central role in many applications (e.g., fluid-structure interaction). The contribution of distributed loads, accounted for at the core-element level, cannot be lifted to the overall level by the projector operator, because the latter brings a nonequilibrated element force vector into equilibrium [7]. To the best of authors' knowledge, this issue has barely been addressed so far in the framework of corotational formulations. Distributed follower loads have been treated in [24], simply leaving the projector operator off the core-element nodal load vector. A rigorous treatment of distributed loads is presented here for the first time, showing that several contributions have to be taken into account in the nodal residual vector and in the consistent tangent stiffness tensor. The analytical derivation of those contributions is carried out in the cases of both dead and follower loads. The resulting formulas for the nodal load vector and for the load tangent stiffness tensor can be implemented with minor computational cost and provide an effective and accurate way to include distributed loads in the analysis. The present treatment can, furthermore, constitute guidance for the rigorous treatment of inertial forces arising in nonlinear dynamic analysis.

The proposed formulation has been validated using popular geometrically nonlinear benchmark problems [25, 26], solved using the full Newton-Raphson method. Comparisons of the present results with those reported in the literature are found to be excellent. Moreover, the present approach compares favorably with standard Lagrangian formulations [25] in terms of consumed iterations.

As an extra benefit for the interested reader, an accessible and adaptable MATLAB toolkit implementing the proposed formulation is provided as supplementary material. It is a front end between the assembler and the core element, and has two main purposes: (1) removing rigid body motions before core-element computations and (2) lifting the nodal residual vector and the consistent tangent stiffness tensor yielded by core-element computations to the overall level. As an example, a combination of the DKT (Discrete Kirchhoff Triangle) plate $[14,27,28]$ and of the optimal ANDES (Assumed Natural DEviatoric Strain) template membrane known as OPT, [29] is used as core element [30]. Of course, any other small-strain or finite-strain triangular shell element with the same node and DOF configuration can be used in the present corotational framework.

The paper is organized as follows. In Section 2, the polar decomposition based corotational kinematics is presented. In Section 3, the equilibrium equations are formulated in such corotational context. Their consistent linearization is dealt with in Section 4. Numerical benchmarks and results are reported and discussed in Section 5. The MATLAB toolkit is briefly described in Appendix A.

\section{A POLAR DECOMPOSITION BASED COROTATIONAL FRAMEWORK}

\subsection{The corotational approach}

The corotational approach is here briefly reviewed through an intrinsic, coordinate-free derivation. Three-node triangular shell elements with six DOFs per node are considered. Let $\boldsymbol{u}_{i}$ and $\boldsymbol{\vartheta}_{i}$, respectively, denote the displacement and rotation vector of the typical node $V_{i}$. The rotation vector $\boldsymbol{\vartheta}_{i}$ is related to the rotation tensor $\boldsymbol{R}_{i}$ by the equation (e.g., [10]):

$$
\boldsymbol{R}_{i}=\exp \left(\operatorname{spin}\left(\boldsymbol{\vartheta}_{i}\right)\right)=\boldsymbol{I}+\frac{\sin \vartheta_{i}}{\vartheta_{i}} \operatorname{spin}\left(\boldsymbol{\vartheta}_{i}\right)+\frac{1-\cos \vartheta_{i}}{\vartheta_{i}^{2}}\left[\operatorname{spin}\left(\boldsymbol{\vartheta}_{i}\right)\right]^{2}
$$

where $\vartheta_{i}=\left\|\vartheta_{i}\right\|, \boldsymbol{I}$ is the identity tensor, and $\|\cdot\|, \operatorname{spin}(\cdot)$, and $\exp (\cdot)$, respectively, denote the norm, the skew-symmetric tensor associated with the argument, and the tensor exponential. In passing, it is recalled that alternative representations of the rotational DOFs, equally suitable for the present derivation, have been put forth in the literature (e.g., [31]). The element nodal parameters $\boldsymbol{u}_{i}, \boldsymbol{\vartheta}_{i}$ are collected into the $18 \times 1$ vector

$$
a=\left\{\boldsymbol{u}_{1} ; \vartheta_{1} ; \boldsymbol{u}_{2} ; \vartheta_{2} ; \boldsymbol{u}_{3} ; \vartheta_{3}\right\}
$$




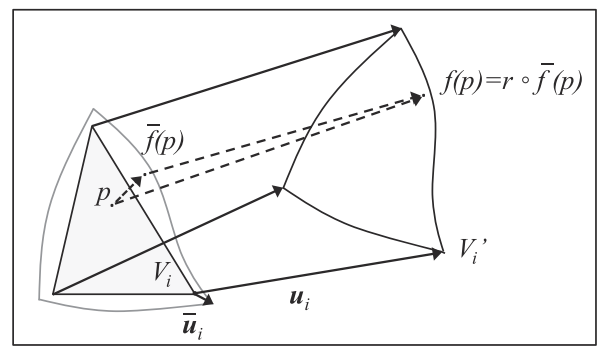

Figure 1. Idea behind the corotational approach.

where the semicolon denotes column stacking. Classical finite element formulations are based on an interpolation of the element displacement field $\boldsymbol{u}$ :

$$
\boldsymbol{u}=\boldsymbol{u}(\boldsymbol{a}, p),
$$

where $p$ is the typical point in the reference configuration.

In the derivation of corotational finite elements, the deformation $f(p)=p+\boldsymbol{u}(p)$ is multiplicatively decomposed as follows (Figure 1):

$$
f=r \circ \bar{f},
$$

where $r$ is a rigid transformation, characterized by a reference point $G$, a translation vector $\boldsymbol{t}$, and a rotation tensor $\boldsymbol{R}$

$$
r(p)=G+\boldsymbol{t}+\boldsymbol{R}[p-G] .
$$

Hence, the transformation $\bar{f}$, implying the same deformational motion as $f$, is obtained from the latter after filtering out the rigid motion $r$. Denoting by $\overline{\boldsymbol{u}}(p)=\bar{f}(p)-p$ the filtered displacement field, Equations (4) and (5) yield

$$
\boldsymbol{u}=G+\boldsymbol{t}+\boldsymbol{R}[p+\overline{\boldsymbol{u}}-G]-p .
$$

Then, a suitable interpolation is chosen for $\overline{\boldsymbol{u}}$, characterizing the core-element formulation

$$
\overline{\boldsymbol{u}}=\overline{\boldsymbol{u}}(\overline{\boldsymbol{a}}, p),
$$

where $\overline{\boldsymbol{a}}$ is the filtered counterpart of the element nodal parameters $\boldsymbol{a}$ (Figure 1)

$$
\overline{\boldsymbol{a}}=\left\{\overline{\boldsymbol{u}}_{1} ; \overline{\boldsymbol{\vartheta}}_{1} ; \overline{\boldsymbol{u}}_{2} ; \overline{\boldsymbol{\vartheta}}_{2} ; \overline{\boldsymbol{u}}_{3} ; \overline{\boldsymbol{\vartheta}}_{3}\right\}
$$

to be used for the computation of the core-element nodal residual vector and of the consistent material tangent stiffness tensor [10]. The filtered nodal displacements $\overline{\boldsymbol{u}}_{i}$ are obtained by imposing the interpolation conditions $\boldsymbol{u}\left(\boldsymbol{a}, V_{i}\right)=\boldsymbol{u}_{i}$ and $\overline{\boldsymbol{u}}\left(\overline{\boldsymbol{a}}, V_{i}\right)=\overline{\boldsymbol{u}}_{i}$ in (6). The following expression is derived:

$$
\overline{\boldsymbol{u}}_{i}=\boldsymbol{R}^{T}\left[V_{i}+\boldsymbol{u}_{i}-(G+\boldsymbol{t})\right]-\left(V_{i}-G\right) .
$$

The filtered nodal rotation vectors $\bar{\vartheta}_{i}$ are given by [7]:

$$
\overline{\boldsymbol{\vartheta}}_{i}=\operatorname{ax} \log \overline{\boldsymbol{R}}_{i},
$$

where $\overline{\boldsymbol{R}}_{i}=\boldsymbol{R}^{T} \boldsymbol{R}_{i}$ are the filtered nodal rotation tensors, ax $(\cdot)$ denotes the axis of a skewsymmetric tensor, and $\log (\cdot)$ denotes the tensor logarithm [32]. In passing, it is noted that the approximation $\overline{\boldsymbol{\vartheta}}_{i} \approx \operatorname{axskw} \overline{\boldsymbol{R}}_{i}$ was proposed in [31] for computational efficiency.

Equations (6)-(10) yield an interpolation recipe for the element displacement field $\boldsymbol{u}$, as a function of the element nodal parameters $\boldsymbol{a}$. As a matter of fact, for a given $\boldsymbol{a}$, one computes $\boldsymbol{t}$ and $\boldsymbol{R}$ as described in Section 2.2, then $\overline{\boldsymbol{a}}$ from (8)-(10), then $\overline{\boldsymbol{u}}$ from (7), and finally $\boldsymbol{u}$ from (6). Hence, 
(6)-(10) can be regarded as the corotational counterpart of (3) and set the corotational approach in the framework of classical finite element procedures. Of course, the dependence of $\boldsymbol{u}$ from $\boldsymbol{a}$ turns out to be highly nonlinear, thus requiring some attention in the computation of the first and second variations (Sections 2.3 and 2.4).

\subsection{The choice of the rigid transformation $r$ : a polar decomposition approach}

Natural choices of the reference point $G$ and of the translation vector $t$ are, respectively, the element centroid and the mean of the element nodal displacements [10]:

$$
G-O=\frac{1}{3} \sum_{i=1}^{3}\left(V_{i}-O\right), \quad \boldsymbol{t}=\frac{1}{3} \sum_{i=1}^{3} \boldsymbol{u}_{i},
$$

where $O$ is a reference point.

The choice of the rotation tensor $\boldsymbol{R}$ as a function of the nodal parameters $\boldsymbol{a}$ is a more delicate issue. In this work, an approach based on polar decomposition is followed [11,12,23]. In particular, $\boldsymbol{R}$ is chosen as the rotation tensor resulting from the polar decomposition of the gradient $\boldsymbol{F}^{\mathrm{h}}$ of the homogeneous deformation mapping the reference triangle $V_{1} V_{2} V_{3}$ onto the triangle $V_{1}^{\prime} V_{2}^{\prime} V_{3}^{\prime}$, where $V_{i}^{\prime}=V_{i}+\boldsymbol{u}_{i}$ (Figure 2(a)). Accordingly, the rotation tensor $\boldsymbol{R}$ turns out to be the best approximation in the Frobenius norm of the deformation gradient $\boldsymbol{F}^{\mathrm{h}}$ [33]. The latter is represented as follows:

$$
\boldsymbol{F}^{\mathrm{h}}=\boldsymbol{J}^{\prime} \boldsymbol{J}^{-1},
$$

where, denoting by $(\xi, \eta)$ an orthonormal basis aligned to the parent element, and by $\otimes$ the tensor product,

$$
\boldsymbol{J}=\left(V_{2}-V_{1}\right) \otimes \xi+\left(V_{3}-V_{1}\right) \otimes \eta, \quad \boldsymbol{J}^{\prime}=\left(V_{2}^{\prime}-V_{1}^{\prime}\right) \otimes \xi+\left(V_{3}^{\prime}-V_{1}^{\prime}\right) \otimes \eta .
$$

For convenience, two orthonormal frames, $\{\boldsymbol{e}, \boldsymbol{h}, \boldsymbol{n}\}$ and $\left\{\boldsymbol{e}^{\prime}, \boldsymbol{h}^{\prime}, \boldsymbol{n}^{\prime}\right\}$, respectively attached to the triangles $V_{1} V_{2} V_{3}$ and $V_{1}^{\prime} V_{2}^{\prime} V_{3}^{\prime}$, are introduced (Figure 2(a)). The former is defined as follows:

$$
\boldsymbol{e}=\frac{V_{2}-V_{1}}{\ell_{12}}, \quad \boldsymbol{h}=\boldsymbol{n} \times \boldsymbol{e}, \quad \boldsymbol{n}=\frac{\boldsymbol{e} \times\left(V_{3}-V_{1}\right)}{\left\|\boldsymbol{e} \times\left(V_{3}-V_{1}\right)\right\|},
$$

where $\ell_{i j}=\left\|V_{i}-V_{j}\right\|$. Analogous definitions, involving symbols affected by an apex, refer to the triangle $V_{1}^{\prime} V_{2}^{\prime} V_{3}^{\prime}$. In particular, it is easily verified that

$$
\begin{array}{r}
\boldsymbol{J}=\ell_{12} \boldsymbol{e} \otimes \xi+\left(\frac{\ell_{12}^{2}+\ell_{31}^{2}-\ell_{23}^{2}}{2 \ell_{12}} \boldsymbol{e}+\frac{2 A}{\ell_{12}} \boldsymbol{h}\right) \otimes \boldsymbol{\eta}, \\
\boldsymbol{J}^{-1}=\xi \otimes\left(\frac{1}{\ell_{12}} \boldsymbol{e}-\frac{\ell_{12}^{2}+\ell_{31}^{2}-\ell_{23}^{2}}{4 A \ell_{12}} \boldsymbol{h}\right)+\boldsymbol{\eta} \otimes \frac{\ell_{12}}{2 A} \boldsymbol{h},
\end{array}
$$

where $A$ denotes the area of the reference triangle $V_{1} V_{2} V_{3}$. [14]:

As pictured in Figure 2(b)-(d), the polar decomposition of $\boldsymbol{F}^{\mathrm{h}}$ is computed in two steps as follows

$$
\boldsymbol{F}^{\mathrm{h}}=\boldsymbol{R} \boldsymbol{U}^{\mathrm{h}}=\hat{\boldsymbol{R}} \check{\boldsymbol{R}} \boldsymbol{U}^{\mathrm{h}} .
$$

Here, $\hat{\boldsymbol{R}}$ is the rotation tensor mapping the frame $\{\boldsymbol{e}, \boldsymbol{h}, \boldsymbol{n}\}$ onto the frame $\left\{\boldsymbol{e}^{\prime}, \boldsymbol{h}^{\prime}, \boldsymbol{n}^{\prime}\right\}$, that is,

$$
\hat{\boldsymbol{R}}=\boldsymbol{e}^{\prime} \otimes \boldsymbol{e}+\boldsymbol{h}^{\prime} \otimes \boldsymbol{h}+\boldsymbol{n}^{\prime} \otimes \boldsymbol{n},
$$

and $\check{\boldsymbol{R}} \boldsymbol{U}^{\mathrm{h}}$ is the polar decomposition of the tensor $\hat{\boldsymbol{R}}^{T} \boldsymbol{F}^{\mathrm{h}}$, denoted by $\hat{\boldsymbol{F}}^{\mathrm{h}}$ in the following. It is emphasized that the latter is the gradient of an in-plane deformation, because $\hat{\boldsymbol{R}}^{T}$ maps the triangle $V_{1}^{\prime} V_{2}^{\prime} V_{3}^{\prime}$ onto the plane of the reference triangle $V_{1} V_{2} V_{3}$ with alignment of one side. From (12) and (13), it turns out that

$$
\hat{\boldsymbol{F}}^{\mathrm{h}}=\hat{\boldsymbol{R}}^{T} \boldsymbol{F}^{\mathrm{h}}=\hat{\boldsymbol{R}}^{T} \boldsymbol{J}^{\prime} \boldsymbol{J}^{-1}=\hat{\boldsymbol{R}}^{T}\left(V_{2}^{\prime}-V_{1}^{\prime}\right) \otimes \boldsymbol{J}^{-T} \xi+\hat{\boldsymbol{R}}^{T}\left(V_{3}^{\prime}-V_{1}^{\prime}\right) \otimes \boldsymbol{J}^{-T} \eta .
$$




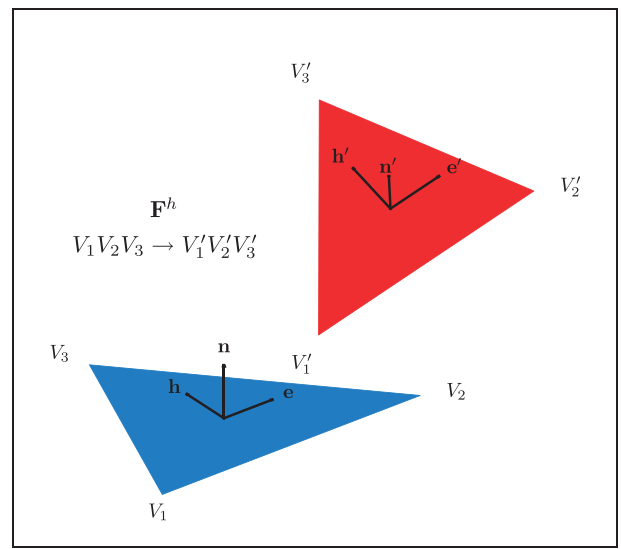

(a)

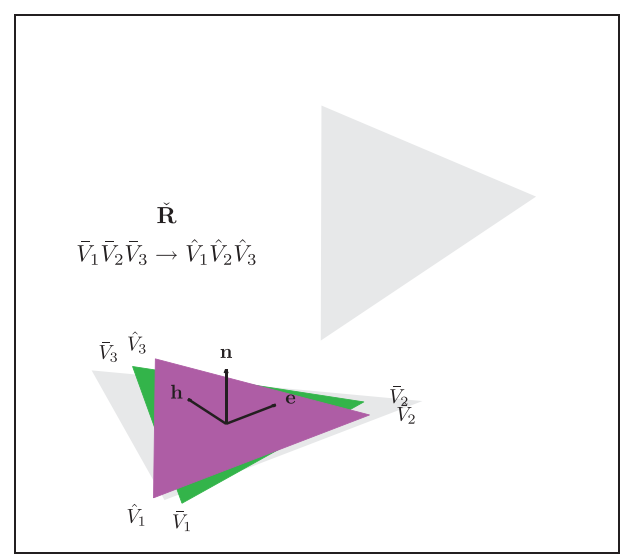

(c)

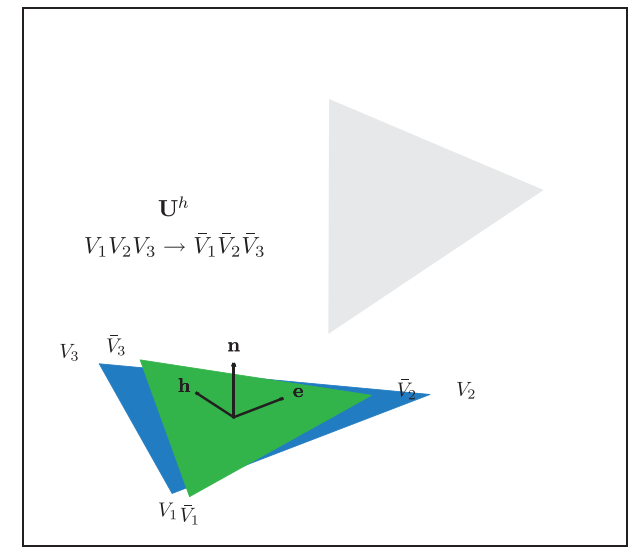

(b)

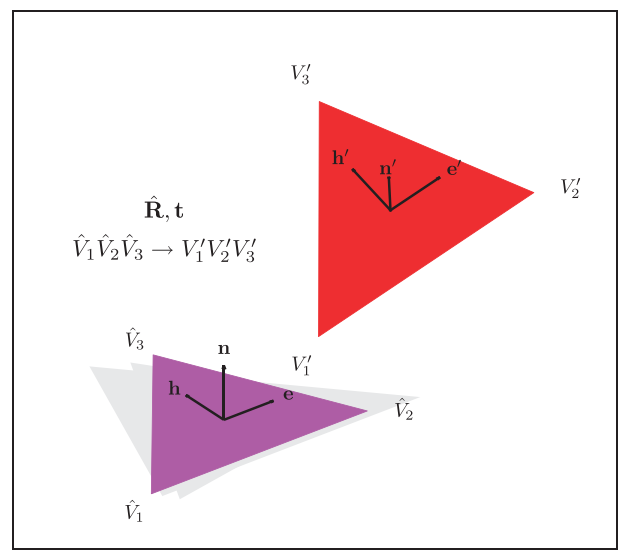

(d)

Figure 2. (a) Homogeneous deformation mapping the reference triangle $V_{1} V_{2} V_{3}$ onto the triangle $V_{1}^{\prime} V_{2}^{\prime} V_{3}^{\prime}$, with gradient $\boldsymbol{F}^{\mathrm{h}}$; (b)-(d) polar decomposition $\boldsymbol{F}^{\mathrm{h}}=\hat{\boldsymbol{R}} \check{\boldsymbol{R}} \boldsymbol{U}^{\mathrm{h}}$ : (b) in-plane stretching $\boldsymbol{U}^{\mathrm{h}}$ of the reference triangle, resulting into $\bar{V}_{1} \bar{V}_{2} \bar{V}_{3}$; (c) in-plane rotation $\check{\boldsymbol{R}}$ of the triangle $\bar{V}_{1} \bar{V}_{2} \bar{V}_{3}$, resulting into $\hat{V}_{1} \hat{V}_{2} \hat{V}_{3}$, which is side aligned with $V_{1} V_{2} V_{3}$; and (d) rototranslation $(\hat{\boldsymbol{R}}, \boldsymbol{t})$ of the triangle $\hat{V}_{1} \hat{V}_{2} \hat{V}_{3}$, returning $V_{1}^{\prime} V_{2}^{\prime} V_{3}^{\prime}$.

Hence, using (15), a simple algebra yields

$$
\check{\boldsymbol{R}}=\cos \alpha(\boldsymbol{e} \otimes \boldsymbol{e}+\boldsymbol{h} \otimes \boldsymbol{h})+\sin \alpha(\boldsymbol{h} \otimes \boldsymbol{e}-\boldsymbol{e} \otimes \boldsymbol{h})+\boldsymbol{n} \otimes \boldsymbol{n},
$$

where

$$
\begin{aligned}
\cos \alpha & =\frac{A\left(\ell_{12}^{\prime}\right)^{2}+A^{\prime} \ell_{12}^{2}}{A \ell_{12} \ell_{12}^{\prime} \operatorname{Tr} \boldsymbol{U}^{\mathrm{h}}}, \quad \sin \alpha=\frac{\ell_{12}^{2}\left[\left(\ell_{23}^{\prime}\right)^{2}-\left(\ell_{31}^{\prime}\right)^{2}\right]+\left(\ell_{12}^{\prime}\right)^{2}\left(\ell_{31}^{2}-\ell_{23}^{2}\right)}{4 A \ell_{12} \ell_{12}^{\prime} \operatorname{Tr} \boldsymbol{U}^{\mathrm{h}}}, \\
\operatorname{Tr} \boldsymbol{U}^{\mathrm{h}} & =\sqrt{\frac{2 A^{\prime}}{A}+\frac{\sum_{k=1}^{3} c_{k}}{8 A^{2}},} \quad c_{k}=\left(\ell_{j k}^{2}+\ell_{i k}^{2}-\ell_{i j}^{2}\right)\left(\ell_{i j}^{\prime}\right)^{2} .
\end{aligned}
$$

Here, $A^{\prime}$ denotes the area of the triangle $V_{1}^{\prime} V_{2}^{\prime} V_{3}^{\prime}$, Tr denotes the trace, and $\{i, j, k\}$ is a cyclic permutation of $\{1,2,3\}$.

According to (16), the rigid transformation $r$ is split into two steps: a first rotation $\check{\boldsymbol{R}}$ followed by a rototranslation $(\hat{\boldsymbol{R}}, \boldsymbol{t})$, both with reference point $G$. Hence, (6) specifies as

$$
\hat{\boldsymbol{u}}=G+\check{\boldsymbol{R}}[p+\overline{\boldsymbol{u}}-G]-p,
$$




$$
\boldsymbol{u}=G+\boldsymbol{t}+\hat{\boldsymbol{R}}[p+\hat{\boldsymbol{u}}-G]-p .
$$

By setting $p=V_{i}, \hat{\boldsymbol{u}}\left(\boldsymbol{a}, V_{i}\right)=\hat{\boldsymbol{u}}_{i}$, and rearranging the terms, (22) and (21), respectively, yield

$$
\begin{gathered}
\hat{\boldsymbol{u}}_{i}=\hat{\boldsymbol{R}}^{\mathrm{T}}\left[V_{i}+\boldsymbol{u}_{i}-(G+\boldsymbol{t})\right]-\left(V_{i}-G\right), \\
\overline{\boldsymbol{u}}_{i}=\check{\boldsymbol{R}}^{\mathrm{T}}\left[V_{i}+\hat{\boldsymbol{u}}_{i}-G\right]-\left(V_{i}-G\right) .
\end{gathered}
$$

Hence, $\hat{\boldsymbol{u}}_{i}$ (respectively, $\overline{\boldsymbol{u}}_{i}$ ) are the nodal displacement vectors resulting after the rototranslation $(\hat{\boldsymbol{R}}, \boldsymbol{t})$ (respectively, the rotation $\check{\boldsymbol{R}}$ ) has been filtered out from $\boldsymbol{u}_{i}$ (respectively, $\hat{\boldsymbol{u}}_{i}$ ). The associated nodal rotation tensors $\hat{\boldsymbol{R}}_{i}$ (respectively, $\overline{\boldsymbol{R}}_{i}$ ), which are the filtered counterparts of $\boldsymbol{R}_{i}$ (respectively, $\left.\hat{\boldsymbol{R}}_{i}\right)$, are given by

$$
\begin{gathered}
\hat{\boldsymbol{R}}_{i}=\hat{\boldsymbol{R}}^{T} \boldsymbol{R}_{i} \\
\text { (respectively, } \left.\overline{\boldsymbol{R}}_{i}=\check{\boldsymbol{R}}^{T} \hat{\boldsymbol{R}}_{i}=\boldsymbol{R}^{T} \boldsymbol{R}_{i}\right) .
\end{gathered}
$$

In summary, recalling (16) and setting $\bar{V}_{i}=V_{i}+\overline{\boldsymbol{u}}_{i}, \hat{V}_{i}=V_{i}+\hat{\boldsymbol{u}}_{i}$, the triangle $V_{1} V_{2} V_{3}$ is mapped by the homogeneous pure deformation $\boldsymbol{U}^{\mathrm{h}}$ onto $\bar{V}_{1} \bar{V}_{2} \bar{V}_{3}$, which is transformed by the rotation $\check{\boldsymbol{R}}$ into $\hat{V}_{1} \hat{V}_{2} \hat{V}_{3}$, side aligned with $V_{1} V_{2} V_{3}$, which in turn is mapped onto $V_{1}^{\prime} V_{2}^{\prime} V_{3}^{\prime}$ by the rototranslation $(\hat{\boldsymbol{R}}, \boldsymbol{t})$ (Figure 2). Accordingly, $\hat{\boldsymbol{F}}^{\mathrm{h}}$ in (18) is recast as follows for a later usage:

$$
\hat{\boldsymbol{F}}^{\mathrm{h}}=\left(\hat{V}_{2}-\hat{V}_{1}\right) \otimes \boldsymbol{J}^{-T} \xi+\left(\hat{V}_{3}-\hat{V}_{1}\right) \otimes \boldsymbol{J}^{-T} \eta .
$$

Moreover, setting $\overline{\boldsymbol{F}}^{\mathrm{h}}=\check{\boldsymbol{R}}^{T} \hat{\boldsymbol{F}}^{\mathrm{h}}=\boldsymbol{R}^{T} \boldsymbol{F}^{\mathrm{h}}$, from (16), it turns out that

$$
\overline{\boldsymbol{F}}^{\mathrm{h}}=\boldsymbol{U}^{\mathrm{h}} \in \mathrm{Sym},
$$

where Sym is the space of symmetric tensors.

\subsection{First variations}

The weak formulation of the equilibrium equations (Section 3) requires the relationship between the variation of the filtered nodal parameters listed in (8), that is,

$$
\delta \overline{\boldsymbol{a}}=\left\{\delta \overline{\boldsymbol{u}}_{1} ; \delta \overline{\boldsymbol{\vartheta}}_{1} ; \delta \overline{\boldsymbol{u}}_{2} ; \delta \overline{\boldsymbol{\vartheta}}_{2} ; \delta \overline{\boldsymbol{u}}_{3} ; \delta \overline{\boldsymbol{\vartheta}}_{3}\right\},
$$

and the variation of the nodal parameters listed in (2), that is,

$$
\delta \boldsymbol{a}=\left\{\delta \boldsymbol{u}_{1} ; \delta \boldsymbol{\vartheta}_{1} ; \delta \boldsymbol{u}_{2} ; \delta \boldsymbol{\vartheta}_{2} ; \delta \boldsymbol{u}_{3} ; \delta \boldsymbol{\vartheta}_{3}\right\} .
$$

Its derivation involves several steps, reported in the succeeding sections. Pivotal equations are highlighted with a box.

2.3.1. From variations of nodal rotation vectors to nodal spins. The nodal spins, defined by

$$
\delta \boldsymbol{\omega}_{i}=\operatorname{ax}\left(\delta \boldsymbol{R}_{i} \boldsymbol{R}_{i}^{T}\right)
$$

are related to the variation of the nodal rotation vectors by

$$
\delta \boldsymbol{\omega}_{i}=\boldsymbol{M}_{i} \delta \boldsymbol{\vartheta}_{i},
$$

where $\boldsymbol{M}_{i}$ is the $3 \times 3$ tensor (e.g., [34])

$$
\boldsymbol{M}_{i}=\boldsymbol{I}+\frac{1-\cos \vartheta_{i}}{\vartheta_{i}^{2}} \operatorname{spin}\left(\boldsymbol{\vartheta}_{i}\right)+\frac{\vartheta_{i}-\sin \vartheta_{i}}{\vartheta_{i}^{3}}\left[\operatorname{spin}\left(\vartheta_{i}\right)\right]^{2} .
$$


The quantities $\delta \boldsymbol{\omega}_{i}$ are collected together with the variations of the nodal displacements $\delta \boldsymbol{u}_{i}$ into the vector

$$
\delta \tilde{\boldsymbol{a}}=\left\{\delta \boldsymbol{u}_{1} ; \delta \boldsymbol{\omega}_{1} ; \delta \boldsymbol{u}_{2} ; \delta \boldsymbol{\omega}_{2} ; \delta \boldsymbol{u}_{3} ; \delta \boldsymbol{\omega}_{3}\right\} .
$$

Hence, the following relationship is derived

$$
\delta \tilde{\boldsymbol{a}}=\boldsymbol{M} \delta \boldsymbol{a},
$$

where

$$
\boldsymbol{M}=\operatorname{diag}\left[\boldsymbol{I}, \boldsymbol{M}_{1}, \boldsymbol{I}, \boldsymbol{M}_{2}, \boldsymbol{I}, \boldsymbol{M}_{3}\right],
$$

and diag denotes the block diagonal assembly of the listed tensors.

2.3.2. Filtering out the rototranslation $(\hat{\boldsymbol{R}}, \boldsymbol{t})$. By differentiating (22), it turns out that

$$
\delta \boldsymbol{u}=\hat{\boldsymbol{R}}[\delta \boldsymbol{\tau}+\delta \hat{\boldsymbol{\theta}} \times(p+\hat{\boldsymbol{u}}-G)+\delta \hat{\boldsymbol{u}}],
$$

where

$$
\begin{gathered}
\delta \boldsymbol{\tau}=\hat{\boldsymbol{R}}^{T} \delta \boldsymbol{t}, \\
\delta \hat{\boldsymbol{\theta}}=\operatorname{ax}\left(\hat{\boldsymbol{R}}^{T} \delta \hat{\boldsymbol{R}}\right) .
\end{gathered}
$$

Two $3 \times 18$ tensors, $\boldsymbol{T}$ and $\hat{\boldsymbol{G}}$, can be derived such that

$$
\begin{gathered}
\delta \boldsymbol{\tau}=\boldsymbol{T}^{\#} \hat{\boldsymbol{R}}^{T} \delta \tilde{\boldsymbol{a}}, \\
\delta \hat{\boldsymbol{\theta}}=\hat{\boldsymbol{G}}^{\#} \hat{\boldsymbol{R}}^{T} \delta \tilde{\boldsymbol{a}},
\end{gathered}
$$

where ${ }^{\sharp} \hat{\boldsymbol{R}}^{T}$ is the block diagonal tensor collecting six copies of $\hat{\boldsymbol{R}}^{T}$. Of course, $\boldsymbol{T}$ and $\hat{\boldsymbol{G}}$ depend on the choice of the translation vector $\boldsymbol{t}$ and of the rotation tensor $\hat{\boldsymbol{R}}$, respectively. From (11), (38), or (17), (39), respectively, they take the form [10]:

$$
\begin{gathered}
\boldsymbol{T}=\frac{1}{3}[\boldsymbol{I}|\mathbf{0}| \boldsymbol{I}|\mathbf{0}| \boldsymbol{I} \mid \mathbf{0}], \\
\hat{\boldsymbol{G}}=\hat{\Xi} \hat{\boldsymbol{Y}} .
\end{gathered}
$$

Here, $\mathbf{0}$ denotes the null tensor, the separator symbol denotes row concatenation, $\hat{\Xi}$ is the $3 \times 3$ tensor

$$
\hat{\Xi}=-\frac{\ell_{12}^{\prime}}{4 A^{\prime}} \boldsymbol{e} \otimes \boldsymbol{e}+\frac{1}{\ell_{12}^{\prime}}(\boldsymbol{h} \otimes \boldsymbol{h}+\boldsymbol{n} \otimes \boldsymbol{n})+\frac{\left(\ell_{31}^{\prime}\right)^{2}-\left(\ell_{23}^{\prime}\right)^{2}}{4 \ell_{12}^{\prime} A^{\prime}} \boldsymbol{e} \otimes \boldsymbol{h},
$$

and $\hat{\boldsymbol{Y}}$ is the $3 \times 18$ tensor

$$
\hat{\boldsymbol{Y}}=[\boldsymbol{e} \otimes \boldsymbol{n}+(\boldsymbol{h} \otimes \boldsymbol{n}-\boldsymbol{n} \otimes \boldsymbol{h})|\mathbf{0}| \boldsymbol{e} \otimes \boldsymbol{n}-(\boldsymbol{h} \otimes \boldsymbol{n}-\boldsymbol{n} \otimes \boldsymbol{h})|\mathbf{0}|-2 \boldsymbol{e} \otimes \boldsymbol{n} \mid \mathbf{0}] .
$$

It is pointed out that $\hat{\boldsymbol{G}}$, known as the spin-fitter tensor, satisfies the geometric separability condition (43), wherein $\hat{\boldsymbol{Y}}$ is independent from the nodal parameters $\boldsymbol{a}$ and $\hat{\boldsymbol{\Xi}}$ is invertible [10]. That condition simplifies the achievement of a consistent linearization, as shown in Section 2.4.

By taking the variation of (23) and using (38) and (39), it turns out that

$$
\delta \hat{\boldsymbol{u}}_{i}=\hat{\boldsymbol{R}}^{T} \delta \boldsymbol{u}_{i}-\left[\delta \boldsymbol{\tau}+\delta \hat{\boldsymbol{\theta}} \times\left(V_{i}+\hat{\boldsymbol{u}}_{i}-G\right)\right]=\hat{\boldsymbol{R}}^{T} \delta \boldsymbol{u}_{i}-\left[\delta \boldsymbol{\tau}-\hat{\boldsymbol{A}}_{i} \delta \hat{\boldsymbol{\theta}}\right],
$$


where $\hat{A}_{i}=\operatorname{spin}\left(V_{i}+\hat{\boldsymbol{u}}_{i}-G\right)$. Moreover, introducing the $\hat{\boldsymbol{R}}$-filtered nodal spins

$$
\delta \hat{\boldsymbol{\omega}}_{i}=\operatorname{ax}\left(\delta \hat{\boldsymbol{R}}_{i} \hat{\boldsymbol{R}}_{i}^{T}\right)
$$

from (25) and (31), it follows that

$$
\begin{aligned}
\delta \hat{\boldsymbol{\omega}}_{i} & =\operatorname{ax}\left(\hat{\boldsymbol{R}}^{T} \delta \boldsymbol{R}_{i} \hat{\boldsymbol{R}}_{i}^{T}+\delta \hat{\boldsymbol{R}}^{T} \boldsymbol{R}_{i} \hat{\boldsymbol{R}}_{i}^{T}\right) \\
& =\operatorname{ax}\left[\hat{\boldsymbol{R}}^{T}\left(\delta \boldsymbol{R}_{i} \boldsymbol{R}_{i}^{T}\right) \hat{\boldsymbol{R}}\right]+\operatorname{ax}\left[\left(\hat{\boldsymbol{R}}^{T} \delta \hat{\boldsymbol{R}}\right)^{T}\right]=\hat{\boldsymbol{R}}^{T} \delta \boldsymbol{\omega}_{i}-\delta \hat{\theta} .
\end{aligned}
$$

The quantities $\delta \hat{\boldsymbol{u}}_{i}$ and $\delta \hat{\boldsymbol{\omega}}_{i}$ are collected into the vector

$$
\delta \hat{\boldsymbol{a}}=\left\{\delta \hat{\boldsymbol{u}}_{1} ; \delta \hat{\boldsymbol{\omega}}_{1} ; \delta \hat{\boldsymbol{u}}_{2} ; \delta \hat{\boldsymbol{\omega}}_{2} ; \delta \hat{\boldsymbol{u}}_{3} ; \delta \hat{\boldsymbol{\omega}}_{3}\right\} .
$$

By substituting $\delta \boldsymbol{\tau}$ and $\delta \hat{\boldsymbol{\theta}}$, respectively, from (40) and (41) into (46) and (48), it turns out from (49), that

$$
\delta \hat{\boldsymbol{a}}=\hat{\boldsymbol{P}}^{\#} \hat{\boldsymbol{R}}^{T} \delta \tilde{\boldsymbol{a}},
$$

where the $18 \times 18$ tensor $\hat{\boldsymbol{P}}$ is given by

$$
\hat{P}=I-(L T+\hat{A} \hat{G})
$$

and the $18 \times 3$ tensors $\boldsymbol{L}$ and $\hat{\boldsymbol{A}}$ are defined as

$$
L=[\boldsymbol{I} ; \mathbf{0} ; \boldsymbol{I} ; \mathbf{0} ; \boldsymbol{I} ; \mathbf{0}], \quad \hat{\boldsymbol{A}}=\left[-\hat{\boldsymbol{A}}_{1} ; \boldsymbol{I} ;-\hat{\boldsymbol{A}}_{2} ; \boldsymbol{I} ;-\hat{\boldsymbol{A}}_{3} ; \boldsymbol{I}\right] .
$$

Here, the semicolon between tensors denotes column stacking. The tensor $\hat{\boldsymbol{A}}$ is known as the spin-lever or moment-arm tensor [10].

The tensor $\hat{\boldsymbol{P}}$ plays a central role in the corotational formulation. From a geometric point of view, it eliminates the rigid body components $\delta \boldsymbol{\tau}$ and $\delta \hat{\boldsymbol{\theta}}$ from the incremental displacement vector $\delta \tilde{\boldsymbol{a}}$ pulled back by ${ }^{\#} \hat{\boldsymbol{R}}^{T}$. Using (74), it is straightforward to verify that $\hat{\boldsymbol{P}}$ is a projector; that is, $\hat{\boldsymbol{P}}^{2}=\hat{\boldsymbol{P}}$ [10].

2.3.3. Filtering out the rotation $\check{\boldsymbol{R}}$. Differentiating (21), it turns out that

$$
\delta \hat{\boldsymbol{u}}=\check{\boldsymbol{R}}[\delta \check{\boldsymbol{\theta}} \times(p+\overline{\boldsymbol{u}}-G)+\delta \overline{\boldsymbol{u}}],
$$

where, using (19),

$$
\delta \check{\boldsymbol{\theta}}=\operatorname{ax}\left(\check{\boldsymbol{R}}^{T} \delta \check{\boldsymbol{R}}\right)=\delta \alpha \boldsymbol{n}
$$

A $3 \times 18$ tensor $\check{\boldsymbol{G}}$ is here derived such that

$$
\delta \check{\boldsymbol{\theta}}=\check{\boldsymbol{G}}^{\#} \check{\boldsymbol{R}}^{T} \delta \hat{a},
$$

where ${ }^{\#} \check{\boldsymbol{R}}^{T}$ is the block diagonal tensor collecting six copies of $\check{\boldsymbol{R}}^{T}$. To this end, recalling (28), it follows that

$$
\operatorname{axskw}\left(\delta \check{\boldsymbol{R}}^{T} \hat{\boldsymbol{F}}^{\mathrm{h}}+\check{\boldsymbol{R}}^{T} \delta \hat{\boldsymbol{F}}^{\mathrm{h}}\right)=\operatorname{axskw}\left(\delta \overline{\boldsymbol{F}}^{\mathrm{h}}\right)=\operatorname{axskw}\left(\delta \boldsymbol{U}^{\mathrm{h}}\right)=\mathbf{0},
$$

and hence, from (54),

$$
\operatorname{axskw}\left(\operatorname{spin}(\delta \check{\boldsymbol{\theta}}) \boldsymbol{U}^{\mathrm{h}}\right)=\operatorname{axskw}\left(\check{\boldsymbol{R}}^{T} \delta \hat{\boldsymbol{F}}^{\mathrm{h}}\right)
$$


The left-hand side of this equation, noting that $\boldsymbol{U}^{\mathrm{h}}$ is a symmetric tensor mapping the span of $\boldsymbol{e}, \boldsymbol{h}$ onto itself, is recast as follows:

$$
\operatorname{axskw}\left(\operatorname{spin}(\delta \check{\boldsymbol{\theta}}) \boldsymbol{U}^{\mathrm{h}}\right)=\operatorname{axskw}\left(\operatorname{spin}(\boldsymbol{n}) \boldsymbol{U}^{\mathrm{h}}\right) \delta \alpha=\frac{1}{2}\left(\operatorname{Tr} \boldsymbol{U}^{\mathrm{h}}\right) \boldsymbol{n} \delta \alpha=\frac{1}{2}\left(\operatorname{Tr} \boldsymbol{U}^{\mathrm{h}}\right) \delta \check{\boldsymbol{\theta}} .
$$

The right-hand side of (57), using (27), becomes

$$
\operatorname{axskw}\left(\check{\boldsymbol{R}}^{T} \delta \hat{\boldsymbol{F}}^{\mathrm{h}}\right)=\operatorname{axskw}\left(\check{\boldsymbol{R}}^{T}\left(\delta \hat{\boldsymbol{u}}_{2}-\delta \hat{\boldsymbol{u}}_{1}\right) \otimes \boldsymbol{J}^{-T} \xi+\check{\boldsymbol{R}}^{T}\left(\delta \hat{\boldsymbol{u}}_{3}-\delta \hat{\boldsymbol{u}}_{1}\right) \otimes \boldsymbol{J}^{-T} \eta\right) .
$$

Hence, from (57)-(59), after simple algebra, it follows that

$$
\check{\boldsymbol{G}}=\check{\Xi} \check{\boldsymbol{Y}},
$$

with

$$
\check{\Xi}=\frac{1}{\operatorname{Tr} \boldsymbol{U}^{\mathrm{h}}} \boldsymbol{I},
$$

and

$$
\begin{aligned}
\check{\boldsymbol{Y}} & =\boldsymbol{n} \otimes \check{\boldsymbol{y}}, \quad \check{\boldsymbol{y}}=\frac{1}{2 A}\left[\boldsymbol{v}_{23}|\mathbf{0}| \boldsymbol{v}_{31}|\mathbf{0}| \boldsymbol{v}_{12} \mid \mathbf{0}\right], \\
\boldsymbol{v}_{23} & =V_{2}-V_{3}=\frac{\ell_{12}^{2}-\ell_{31}^{2}+\ell_{23}^{2}}{2 \ell_{12}} \boldsymbol{e}-\frac{2 A}{\ell_{12}} \boldsymbol{h}, \\
\boldsymbol{v}_{31} & =V_{3}-V_{1}=\frac{\ell_{12}^{2}+\ell_{31}^{2}-\ell_{23}^{2}}{2 \ell_{12}} \boldsymbol{e}+\frac{2 A}{\ell_{12}} \boldsymbol{h}, \\
\boldsymbol{v}_{12} & =V_{1}-V_{2}=-\ell_{12} \boldsymbol{e} .
\end{aligned}
$$

As in the previous case, the spin-fitter tensor $\check{\boldsymbol{G}}$ satisfies the geometric separability condition (60), wherein $\breve{Y}$ is independent from the nodal parameters and $\breve{\Xi}$ is invertible.

By taking the variation of (24) and using (54), it turns out that

$$
\delta \overline{\boldsymbol{u}}_{i}=\check{\boldsymbol{R}}^{T} \delta \hat{\boldsymbol{u}}_{i}-\delta \check{\boldsymbol{\theta}} \times\left(V_{i}+\overline{\boldsymbol{u}}_{i}-G\right)=\check{\boldsymbol{R}}^{T} \delta \hat{\boldsymbol{u}}_{i}+\check{\boldsymbol{A}}_{i}[\delta \check{\boldsymbol{\theta}}],
$$

where $\check{\boldsymbol{A}}_{i}=\operatorname{spin}\left(V_{i}+\overline{\boldsymbol{u}}_{i}-G\right)$. Moreover, introducing the $\boldsymbol{R}$-filtered nodal spins

$$
\delta \check{\boldsymbol{\omega}}_{i}=\operatorname{ax}\left(\delta \overline{\boldsymbol{R}}_{i} \overline{\boldsymbol{R}}_{i}^{T}\right),
$$

from (26) and (47), it turns out that

$$
\begin{aligned}
\delta \check{\boldsymbol{\omega}}_{i} & =\operatorname{ax}\left(\check{\boldsymbol{R}}^{T} \delta \hat{\boldsymbol{R}}_{i} \overline{\boldsymbol{R}}_{i}^{T}+\delta \check{\boldsymbol{R}}^{T} \hat{\boldsymbol{R}}_{i} \overline{\boldsymbol{R}}_{i}^{T}\right) \\
& =\operatorname{ax}\left[\check{\boldsymbol{R}}^{T}\left(\delta \hat{\boldsymbol{R}}_{i} \hat{\boldsymbol{R}}_{i}^{T}\right) \check{\boldsymbol{R}}\right]+\operatorname{ax}\left[\left(\check{\boldsymbol{R}}^{T} \delta \check{\boldsymbol{R}}\right)^{T}\right]=\check{\boldsymbol{R}}^{T} \delta \hat{\boldsymbol{\omega}}_{i}-\delta \check{\boldsymbol{\theta}} .
\end{aligned}
$$

The quantities $\delta \overline{\boldsymbol{u}}_{i}$ and $\delta \check{\boldsymbol{\omega}}_{i}$ are collected into the vector

$$
\delta \check{\boldsymbol{a}}=\left\{\delta \overline{\boldsymbol{u}}_{1} ; \delta \check{\boldsymbol{\omega}}_{1} ; \delta \overline{\boldsymbol{u}}_{2} ; \delta \check{\boldsymbol{\omega}}_{2} ; \delta \overline{\boldsymbol{u}}_{3} ; \delta \check{\boldsymbol{\omega}}_{3}\right\} .
$$

Substituting $\delta \check{\boldsymbol{\theta}}$ from (55) into (63) and (65), Equation (66) gives

$$
\delta \check{\boldsymbol{a}}=\check{\boldsymbol{P}}{ }^{\#} \check{\boldsymbol{R}}^{T} \delta \hat{\boldsymbol{a}},
$$

where the $18 \times 18$ tensor $\check{\boldsymbol{P}}$ is given by

$$
\check{P}=I-\check{A} \check{G}
$$


and the $18 \times 3$ spin-lever tensor $\check{A}$ is defined as

$$
\check{A}=\left[-\check{\boldsymbol{A}}_{1} ; \boldsymbol{I} ;-\check{\boldsymbol{A}}_{2} ; \boldsymbol{I} ;-\check{\boldsymbol{A}}_{3} ; \boldsymbol{I}\right] .
$$

Analogously to $\hat{\boldsymbol{P}}$, also $\check{\boldsymbol{P}}$ plays a central role in the corotational formulation: In fact, it eliminates the rigid body component $\delta \check{\boldsymbol{\theta}}$ from the incremental displacement vector $\delta \hat{\boldsymbol{a}}$ pulled back by ${ }^{\#} \check{\boldsymbol{R}}^{T}$. Using (79) and (60)-(62), it can be verified that $\check{\boldsymbol{P}}$ is a projector; that is, $\check{\boldsymbol{P}}^{2}=\check{\boldsymbol{P}}$.

2.3.4. From filtered nodal spins to variations of filtered nodal rotation vectors. Finally, the transformation from the $\boldsymbol{R}$-filtered nodal spins $\delta \check{\boldsymbol{\omega}}_{i}$, appearing in $\delta \check{\boldsymbol{a}}$, to the variation of the filtered nodal rotation vectors $\delta \overline{\boldsymbol{\vartheta}}_{i}$, appearing in $\delta \overline{\boldsymbol{a}}$, is given by [7]:

$$
\delta \overline{\boldsymbol{a}}=\boldsymbol{B} \delta \check{\boldsymbol{a}},
$$

where

$$
\boldsymbol{B}=\operatorname{diag}\left[\boldsymbol{I}, \boldsymbol{B}_{1}, \boldsymbol{I}, \boldsymbol{B}_{2}, \boldsymbol{I}, \boldsymbol{B}_{3}\right],
$$

with

$$
\boldsymbol{B}_{i}=\boldsymbol{I}-\frac{1}{2} \operatorname{spin}\left(\bar{\vartheta}_{i}\right)+\eta_{i}\left[\operatorname{spin}\left(\bar{\vartheta}_{i}\right)\right]^{2}, \quad \eta_{i}=\frac{1-\frac{\bar{\vartheta}_{i}}{2} \cot \left(\frac{\bar{\vartheta}_{i}}{2}\right)}{\bar{\vartheta}_{i}^{2}}, \quad \bar{\vartheta}_{i}=\left\|\bar{\vartheta}_{i}\right\|
$$

The overall relationship relating $\delta \overline{\boldsymbol{a}}$ to $\delta \boldsymbol{a}$ therefore follows:

$$
\delta \overline{\boldsymbol{a}}=\boldsymbol{B} \check{\boldsymbol{P}}^{\#} \check{\boldsymbol{R}}^{T} \hat{\boldsymbol{P}}^{\#} \hat{\boldsymbol{R}}^{T} \boldsymbol{M} \delta \boldsymbol{a} .
$$

Such relationship coincides with the classical corotational relationship (e.g., [7, 10, 31]), up to the fact that two filtering steps (i.e., $\hat{\boldsymbol{P}}^{\#} \hat{\boldsymbol{R}}^{T}$ and $\check{\boldsymbol{P}}^{\#} \check{\boldsymbol{R}}^{T}$ ) appear here instead of one.

\subsection{Second variations}

Second variations with respect to $\boldsymbol{a}$ are needed for deriving the consistent tangent stiffness tensor, required, for example, by Newton's method of solution. The main task is the differentiation of the spin-fitter tensors $\hat{\boldsymbol{G}}, \check{\boldsymbol{G}}$ and of the projectors $\hat{\boldsymbol{P}}, \check{\boldsymbol{P}}$ with respect to $\boldsymbol{a}$.

2.4.1. Computing $\delta \hat{\boldsymbol{G}}$ and $\delta \hat{\boldsymbol{P}}$. The argument in [7] is here briefly recalled. From (43) and (52), the following orthogonality condition can be derived:

$$
\hat{G} \hat{A}=I .
$$

Substituting (43) into (74) and noting that $\hat{\boldsymbol{Y}} \hat{\boldsymbol{A}}$ turns out to be invertible, it results that

$$
\hat{\Xi}(\hat{Y} \hat{A})=I, \quad \hat{\Xi}=(\hat{Y} \hat{A})^{-1}, \quad \hat{G}=(\hat{Y} \hat{A})^{-1} \hat{Y}
$$

The variation of the first of (75), recalling that $\hat{\boldsymbol{Y}}$ is independent from the nodal parameters, yields

$$
(\delta \hat{\Xi}) \hat{\boldsymbol{Y}} \hat{\boldsymbol{A}}+\hat{\Xi} \hat{\boldsymbol{Y}} \delta \hat{\boldsymbol{A}}=0, \quad \delta \hat{\Xi}=-\hat{\Xi} \hat{\boldsymbol{Y}}(\delta \hat{\boldsymbol{A}})(\hat{\boldsymbol{Y}} \hat{\boldsymbol{A}})^{-1} .
$$

Hence, from (43), (76), and (75), it follows that

$$
\delta \hat{\boldsymbol{G}}=(\delta \hat{\Xi}) \hat{\boldsymbol{Y}}=-\hat{\Xi} \hat{\boldsymbol{Y}}(\delta \hat{\boldsymbol{A}})(\hat{\boldsymbol{Y}} \hat{\boldsymbol{A}})^{-1} \hat{\boldsymbol{Y}}=-\hat{\boldsymbol{G}}(\delta \hat{\boldsymbol{A}}) \hat{\boldsymbol{G}}
$$


Then, using (51), (42), (52), and (77), it turns out that

$$
\begin{aligned}
\delta \hat{\boldsymbol{P}} & =-(\delta \hat{\boldsymbol{A}}) \hat{\boldsymbol{G}}-\hat{\boldsymbol{A}} \delta \hat{\boldsymbol{G}}=-(\delta \hat{\boldsymbol{A}}) \hat{\boldsymbol{G}}+\hat{\boldsymbol{A}} \hat{\boldsymbol{G}}(\delta \hat{\boldsymbol{A}}) \hat{\boldsymbol{G}} \\
& =[-\boldsymbol{I}+\hat{\boldsymbol{A}} \hat{\boldsymbol{G}}](\delta \hat{\boldsymbol{A}}) \hat{\boldsymbol{G}}=[-\boldsymbol{L} \boldsymbol{T}-\hat{\boldsymbol{P}}](\delta \hat{\boldsymbol{A}}) \hat{\boldsymbol{G}}=-\hat{\boldsymbol{P}}(\delta \hat{\boldsymbol{A}}) \hat{\boldsymbol{G}} .
\end{aligned}
$$

In fact, $\boldsymbol{T} \delta \hat{\boldsymbol{A}}=-\frac{1}{3} \delta \operatorname{spin} \sum_{i=1}^{3}\left(V_{i}+\hat{\boldsymbol{u}}_{i}-G\right)=\mathbf{0}$ from (23) and (11).

2.4.2. Computing $\delta \check{\boldsymbol{G}}$ and $\delta \check{\boldsymbol{P}}$. A similar argument is here exploited for the computation of $\delta \check{\boldsymbol{G}}$ and $\delta \check{\boldsymbol{P}}$. From (60) and (69), the following orthogonality condition, analogous to (74), can be derived:

$$
\check{\boldsymbol{G}} \check{\boldsymbol{A}}=\boldsymbol{n} \otimes \boldsymbol{n} .
$$

From a geometric point of view, recalling (69) and (55), this equation states that the vector $\delta \check{\boldsymbol{\theta}}$, resulting from a rigid motion with spin $\delta \alpha \boldsymbol{n}$ of the triangle $\hat{V}_{1} \hat{V}_{2} \hat{V}_{3}$, is just $\delta \alpha \boldsymbol{n}$. Using (60)-(62), it turns out that

$$
(\check{\Xi} n \otimes \check{y}) \check{A}=n \otimes n, \quad(\check{y} \cdot \check{A} n) \check{\Xi} n=n, \quad \hat{\boldsymbol{G}}=\frac{1}{\check{y} \cdot \check{A} n} n \otimes \check{y} .
$$

The quantity $\check{\boldsymbol{y}} \cdot \check{\boldsymbol{A}} \boldsymbol{n}$ does not vanish, by the second of (80), and in fact, it turns out that $\check{\boldsymbol{y}} \cdot \check{\boldsymbol{A}} \boldsymbol{n}=$ $\operatorname{Tr} \boldsymbol{U}^{\mathrm{h}}$. Recalling that $\check{\boldsymbol{y}}$ is independent from the nodal parameters, the variation of the first of (80) yields

$$
(\delta \check{\Xi} n \otimes \check{\boldsymbol{y}}) \check{\boldsymbol{A}}+(\check{\Xi} n \otimes \check{\boldsymbol{y}}) \delta \check{\boldsymbol{A}}=0, \quad \delta \check{\Xi} n=-\frac{\check{\boldsymbol{y}} \cdot \delta \check{\boldsymbol{A}} \boldsymbol{n}}{\check{\boldsymbol{y}} \cdot \check{\boldsymbol{A}} \boldsymbol{n}} \mathrm{\Xi} n .
$$

Hence, using (60), (62), (81), and (80), it results that

$$
\begin{aligned}
& \delta \check{\boldsymbol{G}}=-\frac{\check{\boldsymbol{y}} \cdot \delta \check{\boldsymbol{A}} \boldsymbol{n}}{\check{\boldsymbol{y}} \cdot \check{\boldsymbol{A}} \boldsymbol{n}} \boldsymbol{\Xi} \otimes \check{\boldsymbol{y}}=-\left((\delta \check{\boldsymbol{A}})^{T} \check{\boldsymbol{y}} \cdot \check{\Xi} \boldsymbol{n}\right) \check{\Xi} \boldsymbol{n} \otimes \check{\boldsymbol{y}} \\
& =-\left(\check{\Xi} \boldsymbol{n} \otimes(\delta \check{\boldsymbol{A}})^{T} \check{\boldsymbol{y}}\right) \check{\Xi} \boldsymbol{n} \otimes \check{\boldsymbol{y}}=-\check{\boldsymbol{G}}(\delta \check{\boldsymbol{A}}) \check{\boldsymbol{G}} .
\end{aligned}
$$

Then, using (68) and (82), it follows that

$$
\delta \check{\boldsymbol{P}}=-(\delta \check{\boldsymbol{A}}) \check{\boldsymbol{G}}-\check{\boldsymbol{A}} \delta \check{\boldsymbol{G}}=-(\delta \check{\boldsymbol{A}}) \check{\boldsymbol{G}}+\check{\boldsymbol{A}} \check{\boldsymbol{G}}(\delta \check{\boldsymbol{A}}) \check{\boldsymbol{G}}=-\check{\boldsymbol{P}}(\delta \check{\boldsymbol{A}}) \check{\boldsymbol{G}}
$$

\subsection{Basic relationships}

Some elementary relationships [7] are repeatedly used in the derivation of the consistent tangent stiffness tensor. They are collected here for clarity. Let $w$ be a vector, then

$$
\delta \hat{\boldsymbol{R}} \boldsymbol{w}=-\hat{\boldsymbol{R}}(\operatorname{spin} \boldsymbol{w}) \delta \hat{\boldsymbol{\theta}}, \quad \delta \hat{\boldsymbol{R}}^{T} \boldsymbol{w}=\operatorname{spin}\left(\hat{\boldsymbol{R}}^{T} \boldsymbol{w}\right) \delta \hat{\boldsymbol{\theta}} .
$$

Moreover, let $\boldsymbol{v}$ be the collection of vectors $\boldsymbol{v}=\left\{\boldsymbol{f}_{1} ; \boldsymbol{m}_{1} ; \boldsymbol{f}_{2} ; \boldsymbol{m}_{2} ; \boldsymbol{f}_{3} ; \boldsymbol{m}_{3}\right\}$, then

$$
\begin{aligned}
& \delta^{\#} \hat{\boldsymbol{R}} \boldsymbol{v}=-{ }^{\#} \hat{\boldsymbol{R}} \operatorname{Spin}(\boldsymbol{v}) \delta \hat{\boldsymbol{\theta}}, \\
& \delta \hat{\boldsymbol{G}}^{T} \boldsymbol{v}=-\hat{\boldsymbol{G}}^{T}\left[\operatorname{Spin}\left(\hat{\boldsymbol{G}}^{T} \boldsymbol{v}\right)\right]^{T}{ }^{\mathrm{b}} \boldsymbol{I} \delta \hat{\boldsymbol{a}}, \\
& \delta \hat{\boldsymbol{P}}^{T} \boldsymbol{v}=-\hat{\boldsymbol{G}}^{T}\left[\operatorname{Spin}\left(\hat{\boldsymbol{P}}^{T} \boldsymbol{v}\right)\right]^{T}{ }^{\mathrm{b}} \boldsymbol{I} \delta \hat{\boldsymbol{a}},
\end{aligned}
$$

where the operator $\operatorname{Spin}(\cdot)$ is defined by

$$
\operatorname{Spin}(\boldsymbol{v})=\left[\operatorname{spin}\left(\boldsymbol{f}_{1}\right) ; \operatorname{spin}\left(\boldsymbol{m}_{1}\right) ; \operatorname{spin}\left(\boldsymbol{f}_{2}\right) ; \operatorname{spin}\left(\boldsymbol{m}_{2}\right) ; \operatorname{spin}\left(\boldsymbol{f}_{3}\right) ; \operatorname{spin}\left(\boldsymbol{m}_{3}\right)\right],
$$


and ${ }^{b} \boldsymbol{I}=\operatorname{diag}[\boldsymbol{I}, \mathbf{0}, \boldsymbol{I}, \mathbf{0}, \boldsymbol{I}, \mathbf{0}]$. Equations (84) and the first of (85) follow from (39). The other relationships in (85) follow, respectively, from (77) and (78).

Counterparts of (84) and (85) hold by replacing the hat symbol with the check symbol, and follow from (54), (82), and (83).

\section{EQUILIBRIUM WEAK FORMULATION}

The weak formulation of the equilibrium equations at the element level in a material description is

$$
\boldsymbol{q} \cdot \delta \boldsymbol{a}=\boldsymbol{q}^{\mathrm{i}} \cdot \delta \boldsymbol{a}+\boldsymbol{q}^{1} \cdot \delta \boldsymbol{a}=\int_{\mathcal{V}} \boldsymbol{S}: \delta \boldsymbol{E} \mathrm{d} V-\int_{\mathcal{A}} \boldsymbol{l} \cdot \delta \boldsymbol{u} \mathrm{d} A,
$$

where $\boldsymbol{q}$ is the nodal residual vector and $\boldsymbol{q}^{\mathrm{i}}$ and $\boldsymbol{q}^{1}$ are the nodal internal-force and load vectors, respectively. Moreover, $\mathcal{V}$ and $\mathcal{A}$ denote the element volume and midsurface in the reference configuration, respectively; $\boldsymbol{S}$ is the second Piola-Kirchhoff stress tensor; $\boldsymbol{E}=\left(\boldsymbol{F}^{T} \boldsymbol{F}-\boldsymbol{I}\right) / 2$ is the Green-Lagrange strain tensor; $\boldsymbol{F}=\nabla_{p} f$ is the deformation gradient; $\boldsymbol{l}$ denotes the applied forces per unit reference area, assumed to act on the midsurface. Applied boundary forces on $\partial \mathcal{A}$, if present, should also be added to the right-hand side of (87) and can be treated analogously.

\subsection{Internal-force contribution}

A hyperelastic material is considered, whose constitutive behavior is defined by means of the strain energy function $W(\boldsymbol{E})$, so that the stress-strain relationship is $\boldsymbol{S}(\boldsymbol{E})=\partial_{\boldsymbol{E}} \mathrm{W}(\boldsymbol{E})$. From (4) and (5), it turns out that $\boldsymbol{F}=\boldsymbol{R} \overline{\boldsymbol{F}}$, where $\overline{\boldsymbol{F}}=\nabla_{p} \bar{f}$ is the filtered deformation gradient. Hence, the filtered Green-Lagrange strain tensor $\overline{\boldsymbol{E}}=\left(\overline{\boldsymbol{F}}^{T} \overline{\boldsymbol{F}}-\boldsymbol{I}\right) / 2$ coincides with $\boldsymbol{E}$. As a consequence, the internal-force contribution is recast as

$$
\boldsymbol{q}^{\mathrm{i}} \cdot \delta \boldsymbol{a}=\int_{\mathcal{V}} \boldsymbol{S}(\boldsymbol{E}): \delta \boldsymbol{E} \mathrm{d} V=\int_{\mathcal{V}} \boldsymbol{S}(\overline{\boldsymbol{E}}): \delta \overline{\boldsymbol{E}} \mathrm{d} V=\overline{\boldsymbol{q}}^{\mathrm{i}} \cdot \delta \overline{\boldsymbol{a}},
$$

where $\overline{\boldsymbol{q}}^{\mathrm{i}}$ is the nodal internal-force vector work-conjugated to the filtered nodal parameters. It is pointed out that the last equality is the core-element equilibrium weak formulation, entailing the interpolation (7) for the relevant finite element implementation. In passing, it is noted that the tensor $\overline{\boldsymbol{F}}$, that is, $\boldsymbol{I}+\nabla_{p} \overline{\boldsymbol{u}}$, should be distinguished from $\overline{\boldsymbol{F}}^{\mathrm{h}}$ defined in (28). Indeed, the former is not generally symmetric, and it would coincide with the latter only in the case of a constant-strain core element.

\subsection{Distributed load contribution}

Two types of load are here considered:

- dead loads $\boldsymbol{l}^{\mathrm{d}}$, defined per unit reference area. An example is self-weight.

- follower loads $\boldsymbol{l}^{\mathrm{f}}$, defined per unit deformed area, rotating element-wise according to the rotation tensor $\boldsymbol{R}$. An example is pressure load.

Hence, the load appearing in (87) is given by

$$
\boldsymbol{l}=\boldsymbol{l}^{\mathrm{d}}+\boldsymbol{R} \boldsymbol{l}^{\mathrm{f}} A^{\prime} / A,
$$

where $\boldsymbol{l}^{\mathrm{d}}$ and $\boldsymbol{l}^{\mathrm{f}}$ are assumed to be independent from the nodal parameters $\boldsymbol{a}$. Exploiting the corotational kinematics (i.e., (37) and (53)), the load contribution in (87) is recast as

$$
\boldsymbol{q}^{1} \cdot \delta a=-\check{\boldsymbol{R}} \boldsymbol{f} \cdot \delta \boldsymbol{\tau}-\check{\boldsymbol{R}} \boldsymbol{m}_{G} \cdot \delta \hat{\boldsymbol{\theta}}-\boldsymbol{m}_{G} \cdot \delta \check{\boldsymbol{\theta}}+\overline{\boldsymbol{q}}^{\mathrm{l}} \cdot \delta \overline{\boldsymbol{a}},
$$


yielding $\boldsymbol{q}^{1}$ in terms of the resultant force and moment of the loads $\boldsymbol{l}$ pulled back by $\boldsymbol{R}^{T}$, that is,

$$
\begin{gathered}
\boldsymbol{f}=\int_{\mathcal{A}}\left(\boldsymbol{R}^{T} \boldsymbol{l}^{\mathrm{d}}+\boldsymbol{l}^{\mathrm{f}} A^{\prime} / A\right) \mathrm{d} A=\boldsymbol{f}^{\mathrm{d}}+\boldsymbol{f}^{\mathrm{f}}, \\
\boldsymbol{m}_{G}=\int_{\mathcal{A}}(p+\overline{\boldsymbol{u}}(p)-G) \times\left(\boldsymbol{R}^{T} \boldsymbol{l}^{\mathrm{d}}+\boldsymbol{l}^{\mathrm{f}} A^{\prime} / A\right) \mathrm{d} A=\boldsymbol{m}_{G}^{\mathrm{d}}+\boldsymbol{m}_{G}^{\mathrm{f}},
\end{gathered}
$$

and in terms of the core-element nodal load vector $\overline{\boldsymbol{q}}^{1}$, defined by

$$
\overline{\boldsymbol{q}}^{\mathrm{l}}=-\int_{\mathcal{A}}\left(\frac{\partial \overline{\boldsymbol{u}}}{\partial \overline{\boldsymbol{a}}}\right)^{T}\left(\boldsymbol{R}^{T} \boldsymbol{l}^{\mathrm{d}}+\boldsymbol{l}^{\mathrm{f}} A^{\prime} / A\right) \mathrm{d} A=\overline{\boldsymbol{q}}^{\mathrm{d}}+\overline{\boldsymbol{q}}^{\mathrm{f}} .
$$

Here, $\partial \overline{\boldsymbol{u}} / \partial \overline{\boldsymbol{a}}$ are the core-element displacement shape functions, depending on the core-element interpolation (7).

Exploiting (40), (41), and (55), Equation (90) is recast as

$$
\boldsymbol{q}^{1} \cdot \delta \boldsymbol{a}=\tilde{\boldsymbol{q}}^{1} \cdot \delta \tilde{\boldsymbol{a}}+\hat{\boldsymbol{q}}^{1} \cdot \delta \hat{\boldsymbol{a}}+\overline{\boldsymbol{q}}^{1} \cdot \delta \overline{\boldsymbol{a}}
$$

where

$$
\tilde{\boldsymbol{q}}^{1}=-{ }^{\#} \hat{\boldsymbol{R}} \boldsymbol{T}^{T} \check{\boldsymbol{R}} \boldsymbol{f}-{ }^{\#} \hat{\boldsymbol{R}} \hat{\boldsymbol{G}}^{T} \check{\boldsymbol{R}} \boldsymbol{m}_{G}, \quad \hat{\boldsymbol{q}}^{1}=-{ }^{\#} \check{\boldsymbol{R}} \check{\boldsymbol{G}}^{T} \boldsymbol{m}_{G} .
$$

\subsection{Nodal residual vector}

From the virtual work equations (87), (88), and (94), it follows that

$$
\boldsymbol{q} \cdot \delta \boldsymbol{a}=\tilde{\boldsymbol{q}}^{1} \cdot \delta \tilde{\boldsymbol{a}}+\hat{\boldsymbol{q}}^{1} \cdot \delta \hat{\boldsymbol{a}}+\left(\overline{\boldsymbol{q}}^{\mathrm{i}}+\overline{\boldsymbol{q}}^{\mathrm{l}}\right) \cdot \delta \overline{\boldsymbol{a}} .
$$

Hence, using (35), (50), (67), and (70), the following algorithm for the computation of the nodal residual vector is obtained:

$$
\begin{aligned}
& \tilde{\boldsymbol{q}}^{1} \cdot \delta \tilde{\boldsymbol{a}}+\hat{\boldsymbol{q}}^{1} \cdot \delta \hat{\boldsymbol{a}}+\underbrace{\left(\overline{\boldsymbol{q}}^{\mathrm{i}}+\overline{\boldsymbol{q}}^{1}\right)} \cdot \delta \overline{\boldsymbol{a}} \stackrel{\downarrow}{=} \delta \overline{\boldsymbol{a}}=\boldsymbol{B} \delta \check{\boldsymbol{a}} \\
& \tilde{\boldsymbol{q}}^{1} \cdot \delta \tilde{\boldsymbol{a}}+\hat{\boldsymbol{q}}^{1} \cdot \delta \hat{\boldsymbol{a}}+\underbrace{\boldsymbol{B}^{T} \overline{\boldsymbol{q}}} \cdot \delta \check{\boldsymbol{a}} \stackrel{\downarrow}{=} \delta \check{\boldsymbol{a}}=\check{\boldsymbol{P}}^{\# \check{\boldsymbol{R}}^{T}} \delta \hat{\boldsymbol{a}} \\
& \tilde{\boldsymbol{q}}^{1} \cdot \delta \tilde{\boldsymbol{a}}+(\underbrace{\hat{\boldsymbol{q}}^{1}+{ }^{\#} \check{\boldsymbol{R}} \check{\boldsymbol{P}}^{T} \check{\boldsymbol{q}}}) \cdot \delta \hat{\boldsymbol{a}} \stackrel{\downarrow}{=} \delta \hat{\boldsymbol{a}}=\hat{\boldsymbol{P}}^{\# \hat{\boldsymbol{R}}^{T} \delta \tilde{\boldsymbol{a}}} \\
& \underbrace{\left(\tilde{\boldsymbol{q}}^{1}+{ }^{\#} \hat{\boldsymbol{R}} \hat{\boldsymbol{P}}^{T} \hat{\boldsymbol{q}}\right)} \cdot \delta \tilde{\boldsymbol{a}} \stackrel{\unrhd}{=} \delta \tilde{\boldsymbol{a}}=\boldsymbol{M} \delta \boldsymbol{a} \\
& \underbrace{\boldsymbol{M}^{T} \tilde{\boldsymbol{q}}} \cdot \delta \boldsymbol{a}= \\
& \boldsymbol{q} \cdot \delta \boldsymbol{a},
\end{aligned}
$$

where the vectors $\overline{\boldsymbol{q}}, \check{\boldsymbol{q}}, \hat{\boldsymbol{q}}$, and $\tilde{\boldsymbol{q}}$, defined by the underbraces, are given by

$$
\overline{\boldsymbol{q}}=\overline{\boldsymbol{q}}^{\mathrm{i}}+\overline{\boldsymbol{q}}^{\mathrm{l}}, \quad \check{\boldsymbol{q}}=\boldsymbol{B}^{T} \overline{\boldsymbol{q}}, \quad \hat{\boldsymbol{q}}=\hat{\boldsymbol{q}}^{1}+{ }^{\#} \check{\boldsymbol{R}} \check{\boldsymbol{P}}^{T} \check{\boldsymbol{q}}, \quad \tilde{\boldsymbol{q}}=\tilde{\boldsymbol{q}}^{1}+{ }^{\#} \hat{\boldsymbol{R}} \hat{\boldsymbol{P}}^{T} \hat{\boldsymbol{q}}, \quad \boldsymbol{q}=\boldsymbol{M}^{T} \tilde{\boldsymbol{q}} .
$$

When no distributed loads are present (i.e., $\boldsymbol{l}^{\mathrm{d}}=\boldsymbol{l}^{\mathrm{f}}=0$, so that $\overline{\boldsymbol{q}}^{1}=\hat{\boldsymbol{q}}^{1}=\tilde{\boldsymbol{q}}^{1}=\mathbf{0}$ ), the algorithms (97) and (98) reduce to

$$
\boldsymbol{q}=\boldsymbol{M}^{T \#} \hat{\boldsymbol{R}} \hat{\boldsymbol{P}}^{T}{ }^{\#} \check{\boldsymbol{R}} \check{\boldsymbol{P}}^{T} \boldsymbol{B}^{T} \overline{\boldsymbol{q}}^{\mathrm{i}},
$$

which has the classical multiplicative structure (e.g., [7]), except that two filtering steps (i.e., ${ }^{\#} \check{\boldsymbol{R}} \check{\boldsymbol{P}}^{T}$ and ${ }^{\#} \hat{\boldsymbol{R}} \hat{\boldsymbol{P}}^{T}$ ) appear here instead of one. 
The presence of applied follower loads was heuristically accounted for in [24]. That approach consists in leaving projectors off (99), 'because rigid body portions of the applied force should not be projected out'. Hence, according to [24], the following term

$$
\boldsymbol{M}^{T \#} \hat{\boldsymbol{R}}^{\#} \check{\boldsymbol{R}} \boldsymbol{B}^{T} \overline{\boldsymbol{q}}^{1}
$$

should be added to the left-hand side of (99). However, the present derivation shows that the proper contribution to thoroughly account for distributed loads is

$$
\boldsymbol{M}^{T}\left(\tilde{\boldsymbol{q}}^{1}+{ }^{\#} \hat{\boldsymbol{R}} \hat{\boldsymbol{P}}^{T}\left(\hat{\boldsymbol{q}}^{1}+{ }^{\#} \check{\boldsymbol{R}} \check{\boldsymbol{P}}^{T} \boldsymbol{B}^{T} \overline{\boldsymbol{q}}^{1}\right)\right),
$$

as follows by comparing (98) with (99).

\section{LINEARIZATION}

The linearization of the virtual work equation (96) is performed as follows:

$$
\begin{aligned}
\Delta(\boldsymbol{q} \cdot \delta \boldsymbol{a})= & \Delta\left(\overline{\boldsymbol{q}}^{\mathrm{i}}+\overline{\boldsymbol{q}}^{\mathrm{l}}\right) \cdot \delta \overline{\boldsymbol{a}}+\Delta \hat{\boldsymbol{q}}^{1} \cdot \delta \hat{\boldsymbol{a}}+\Delta \tilde{\boldsymbol{q}}^{1} \cdot \delta \tilde{\boldsymbol{a}} \\
& +\left(\overline{\boldsymbol{q}}^{\mathrm{i}}+\overline{\boldsymbol{q}}^{1}\right) \cdot \Delta \delta \overline{\boldsymbol{a}}+\hat{\boldsymbol{q}}^{1} \cdot \Delta \delta \hat{\boldsymbol{a}}+\tilde{\boldsymbol{q}}^{1} \cdot \Delta \delta \tilde{\boldsymbol{a}},
\end{aligned}
$$

where $\Delta$ denotes the linearization operator. For clarity, the contributions arising from each term in the right-hand side of (102) are separately dealt with in Sections 4.1-4.5. Then, they are assembled in Section 4.6, where the algorithm for the computation of the consistent tangent stiffness tensor is provided.

\subsection{Contribution from the term involving $\Delta \overline{\boldsymbol{q}}^{i}$}

By definition,

$$
\Delta \overline{\boldsymbol{q}}^{\mathrm{i}} \cdot \delta \overline{\boldsymbol{a}}=\overline{\boldsymbol{K}}_{\overline{\boldsymbol{q}}^{\mathrm{i}}} \Delta \overline{\boldsymbol{a}} \cdot \delta \overline{\boldsymbol{a}},
$$

where $\overline{\boldsymbol{K}}_{\overline{\boldsymbol{q}}^{\mathrm{i}}}=\partial \overline{\boldsymbol{q}}^{\mathrm{i}} / \partial \overline{\boldsymbol{a}}$ is the material tangent stiffness tensor and depends on the core-element formulation.

\subsection{Contribution from the term involving $\Delta \overline{\boldsymbol{q}}^{1}$}

From (93), assuming the displacement shape functions $\partial \overline{\boldsymbol{u}} / \partial \overline{\boldsymbol{a}}$ to be independent from $\overline{\boldsymbol{a}}$, and using the second of (84) and its check counterpart, it turns out that

$$
\Delta \overline{\boldsymbol{q}}^{\mathrm{l}}=\overline{\boldsymbol{Q}}^{\mathrm{d}}\left(\check{\boldsymbol{R}}^{T} \Delta \hat{\boldsymbol{\theta}}+\Delta \check{\boldsymbol{\theta}}\right)+\overline{\boldsymbol{q}}^{\mathrm{f}}(\boldsymbol{b} \cdot \Delta \overline{\boldsymbol{a}}),
$$

where

$$
\overline{\boldsymbol{Q}}^{\mathrm{d}}=-\int_{\mathcal{A}}\left(\frac{\partial \overline{\boldsymbol{u}}}{\partial \overline{\boldsymbol{a}}}\right)^{T} \operatorname{spin}\left(\boldsymbol{R}^{T} \boldsymbol{l}^{\mathrm{d}}\right) \mathrm{d} A,
$$

and, recalling that the vectors $\Delta \overline{\boldsymbol{u}}_{i}$ appearing in $\Delta \overline{\boldsymbol{a}}$ belong to the span of $\boldsymbol{e}, \boldsymbol{h}$,

$$
\boldsymbol{b}=\frac{1}{A^{\prime}} \frac{\partial A^{\prime}}{\partial \overline{\boldsymbol{a}}}=\frac{1}{2 A^{\prime}}\left[\boldsymbol{n} \times\left(\bar{V}_{3}-\bar{V}_{2}\right)|\mathbf{0}| \boldsymbol{n} \times\left(\bar{V}_{1}-\bar{V}_{3}\right)|\mathbf{0}| \boldsymbol{n} \times\left(\bar{V}_{2}-\bar{V}_{1}\right) \mid \mathbf{0}\right] .
$$

Hence, by (55), (41), (70), (67), and (50), it follows that

$$
\Delta \overline{\boldsymbol{q}}^{1} \cdot \delta \overline{\boldsymbol{a}}=\overline{\boldsymbol{K}}_{\overline{\boldsymbol{q}}^{1}} \Delta \overline{\boldsymbol{a}} \cdot \delta \overline{\boldsymbol{a}}+\hat{\boldsymbol{K}}_{\overline{\boldsymbol{q}}^{1}} \Delta \hat{\boldsymbol{a}} \cdot \delta \hat{\boldsymbol{a}}+\tilde{\boldsymbol{K}}_{\overline{\boldsymbol{q}}^{1}} \Delta \tilde{\boldsymbol{a}} \cdot \delta \tilde{\boldsymbol{a}},
$$

with

$$
\overline{\boldsymbol{K}}_{\overline{\boldsymbol{q}}^{1}}=\overline{\boldsymbol{q}}^{\mathrm{f}} \otimes \boldsymbol{b}, \quad \hat{\boldsymbol{K}}_{\overline{\boldsymbol{q}}^{1}}={ }^{\#} \check{\boldsymbol{R}} \check{\boldsymbol{P}}^{T} \boldsymbol{B}^{T} \overline{\boldsymbol{Q}}^{\mathrm{d}} \check{\boldsymbol{G}}^{\#} \check{\boldsymbol{R}}^{T}, \quad \tilde{\boldsymbol{K}}_{\overline{\boldsymbol{q}}^{1}}={ }^{\#} \hat{\boldsymbol{R}} \hat{\boldsymbol{P}}^{T}{ }^{\#} \check{\boldsymbol{R}} \check{\boldsymbol{P}}^{T} \boldsymbol{B}^{T} \overline{\boldsymbol{Q}}^{\mathrm{d}} \check{\boldsymbol{R}}^{T} \check{\boldsymbol{G}}^{\#} \hat{\boldsymbol{R}}^{T} .
$$




\subsection{Contribution from the term involving $\Delta \hat{\boldsymbol{q}}^{1}$}

From (95), it turns out that

$$
\Delta \hat{\boldsymbol{q}}^{1}=-\left(\hat{\boldsymbol{K}}_{\# \check{\boldsymbol{R}} \check{\boldsymbol{G}}^{T}}\left[\boldsymbol{m}_{G}\right] \Delta \hat{\boldsymbol{a}}+{ }^{\#} \check{\boldsymbol{R}} \check{\boldsymbol{G}}^{T} \Delta \boldsymbol{m}_{G}\right),
$$

where

$$
\hat{\boldsymbol{K}}_{\# \check{\boldsymbol{R}} \check{\boldsymbol{G}}}[\boldsymbol{v}] \Delta \hat{\boldsymbol{a}}=\left.\Delta\left({ }^{\#} \check{\boldsymbol{R}} \check{\boldsymbol{G}}^{T} \boldsymbol{v}\right)\right|_{\boldsymbol{v}=\text { const }} .
$$

The tensor $\hat{\boldsymbol{K}}_{\# \check{\boldsymbol{R}} \check{\boldsymbol{G}}^{T}}[\boldsymbol{v}]$ comprises two contributions: a rotational geometric stiffness, related to the linearization of the rotation tensor $\check{\boldsymbol{R}}$, and a spin-fitter geometric stiffness, related to the linearization of the spin-fitter $\check{\boldsymbol{G}}$. Exploiting the check counterparts of (85), (55), and (67), it follows that

$$
\hat{\boldsymbol{K}}_{\# \check{\boldsymbol{R}} \check{\boldsymbol{G}}^{T}}[\boldsymbol{v}]={ }^{\#} \check{\boldsymbol{R}}\left[\operatorname{Spin}\left(\check{\boldsymbol{G}}^{T} \boldsymbol{v}\right) \check{\boldsymbol{G}}+\check{\boldsymbol{G}}^{T}\left[\operatorname{Spin}\left(\check{\boldsymbol{G}}^{T} \boldsymbol{v}\right)\right]^{T}{ }^{\mathrm{b}} \boldsymbol{I} \check{\boldsymbol{P}}\right] \#^{\#} \check{\boldsymbol{R}}^{T} .
$$

From (92), it turns out that

$$
\Delta \boldsymbol{m}_{G}=-\overline{\boldsymbol{Q}}^{T} \Delta \overline{\boldsymbol{a}}+\boldsymbol{M}_{G}^{\mathrm{d}}\left(\check{\boldsymbol{R}}^{T} \Delta \hat{\boldsymbol{\theta}}+\Delta \check{\boldsymbol{\theta}}\right)+\boldsymbol{m}_{G}^{\mathrm{f}}(\boldsymbol{b} \cdot \Delta \overline{\boldsymbol{a}}),
$$

where $\overline{\boldsymbol{Q}}=\overline{\boldsymbol{Q}}^{\mathrm{d}}+\overline{\boldsymbol{Q}}^{\mathrm{f}}$, and

$$
\overline{\boldsymbol{Q}}^{\mathrm{f}}=-\int_{\mathcal{A}}\left(\frac{\partial \overline{\boldsymbol{u}}}{\partial \overline{\boldsymbol{a}}}\right)^{T} \operatorname{spin}\left(\boldsymbol{l}^{\mathrm{f}} A^{\prime} / A\right) \mathrm{d} A, \quad \boldsymbol{M}_{G}^{\mathrm{d}}=\int_{\mathcal{A}} \operatorname{spin}(p+\overline{\boldsymbol{u}}(p)-G) \operatorname{spin}\left(\boldsymbol{R}^{T} \boldsymbol{l}^{\mathrm{d}}\right) \mathrm{d} A .
$$

Hence, by (70), (67), (55), (41), and (50), it turns out that

$$
\Delta \hat{\boldsymbol{q}}^{1} \cdot \delta \hat{\boldsymbol{a}}=\hat{\boldsymbol{K}}_{\hat{\boldsymbol{q}}^{1}} \Delta \hat{\boldsymbol{a}} \cdot \delta \hat{\boldsymbol{a}}+\tilde{\boldsymbol{K}}_{\hat{\boldsymbol{q}}^{1}} \Delta \tilde{\boldsymbol{a}} \cdot \delta \tilde{\boldsymbol{a}},
$$

with

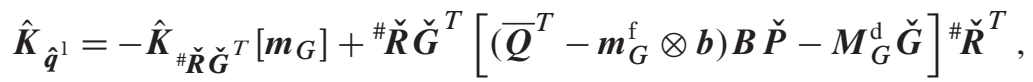

$$
\begin{aligned}
& \hat{\boldsymbol{K}}_{\hat{\boldsymbol{q}}^{1}}=-{ }^{\#} \hat{\boldsymbol{R}} \hat{\boldsymbol{P}}^{T}{ }^{\#} \check{\boldsymbol{R}} \check{\boldsymbol{G}}^{T} \boldsymbol{M}_{G}^{\mathrm{d}} \check{\boldsymbol{R}}^{T} \hat{\boldsymbol{G}}^{\# \hat{\boldsymbol{R}}^{T}} \text {. }
\end{aligned}
$$

\subsection{Contribution from the term involving $\Delta \tilde{\boldsymbol{q}}^{1}$}

From equation (95), noting that no contribution arises from $\boldsymbol{f}^{\mathrm{d}}$ because ${ }^{\#} \hat{\boldsymbol{R}} \boldsymbol{T}^{T}=\boldsymbol{T}^{T} \hat{\boldsymbol{R}}$, and using the first of (84) and its check counterpart, it follows that

$$
\begin{aligned}
& \Delta \tilde{\boldsymbol{q}}^{1}=\boldsymbol{T}^{T} \boldsymbol{R} \operatorname{spin}\left(\boldsymbol{f}^{\mathrm{f}}\right)\left(\check{\boldsymbol{R}}^{T} \Delta \hat{\boldsymbol{\theta}}+\Delta \check{\boldsymbol{\theta}}\right)-\boldsymbol{T}^{T} \boldsymbol{R} \boldsymbol{f}^{\mathrm{f}}(\boldsymbol{b} \cdot \Delta \overline{\boldsymbol{a}}) \\
& -\tilde{\boldsymbol{K}}_{\# \hat{\boldsymbol{R}} \hat{\boldsymbol{G}}^{T}}\left[\check{\boldsymbol{R}} \boldsymbol{m}_{G}\right] \Delta \tilde{\boldsymbol{a}}+{ }^{\#} \hat{\boldsymbol{R}} \hat{\boldsymbol{G}}^{T}\left[\check{\boldsymbol{R}} \operatorname{spin}\left(\boldsymbol{m}_{G}\right) \Delta \check{\boldsymbol{\theta}}-\check{\boldsymbol{R}} \Delta \boldsymbol{m}_{G}\right],
\end{aligned}
$$

where

$$
\tilde{\boldsymbol{K}}_{\# \hat{\boldsymbol{R}} \hat{\boldsymbol{G}}^{T}}[\boldsymbol{v}] \Delta \tilde{\boldsymbol{a}}=\left.\Delta\left({ }^{\#} \hat{\boldsymbol{R}} \hat{\boldsymbol{G}}^{T} \boldsymbol{v}\right)\right|_{\boldsymbol{v}=\text { const }} .
$$

The tensor $\tilde{\boldsymbol{K}}_{\# \hat{\boldsymbol{R}} \hat{\boldsymbol{G}}^{T}}[\boldsymbol{v}]$ is the counterpart of the tensor $\hat{\boldsymbol{K}}_{\# \check{\boldsymbol{R}} \check{\boldsymbol{G}}^{T}}[\boldsymbol{v}]$ defined in (111) and, exploiting (85), (41), and (50), it takes the form

$$
\tilde{\boldsymbol{K}}_{\# \hat{\boldsymbol{R}} \hat{\boldsymbol{G}}^{T}}[\boldsymbol{v}]=-{ }^{\#} \hat{\boldsymbol{R}}\left[\operatorname{Spin}\left(\hat{\boldsymbol{G}}^{T} \boldsymbol{v}\right) \hat{\boldsymbol{G}}+\hat{\boldsymbol{G}}^{T}\left[\operatorname{Spin}\left(\hat{\boldsymbol{G}}^{T} \boldsymbol{v}\right)\right]^{T}{ }^{\mathrm{I}} \boldsymbol{\boldsymbol { P }}\right]{ }^{\#} \hat{\boldsymbol{R}}^{T} .
$$

Hence, by (41), (55), (50), (112), (70), and (67), it turns out that

$$
\Delta \tilde{\boldsymbol{q}}^{1} \cdot \delta \tilde{\boldsymbol{a}}=\tilde{\boldsymbol{K}}_{\tilde{\boldsymbol{q}}^{1}} \Delta \tilde{\boldsymbol{a}} \cdot \delta \tilde{\boldsymbol{a}},
$$


with

$$
\begin{aligned}
& \tilde{\boldsymbol{K}}_{\tilde{\boldsymbol{q}}^{1}}=\boldsymbol{T}^{T} \boldsymbol{R}\left[\operatorname{spin}\left(\boldsymbol{f}^{\mathrm{f}}\right)\left(\check{\boldsymbol{R}}^{T} \hat{\boldsymbol{G}}+\check{\boldsymbol{G}}^{\#} \check{\boldsymbol{R}}^{T} \hat{\boldsymbol{P}}\right)-\left(\boldsymbol{f}^{\mathrm{f}} \otimes \boldsymbol{b}\right) \boldsymbol{B} \check{\boldsymbol{P}}^{\#} \check{\boldsymbol{R}}^{T} \hat{\boldsymbol{P}}\right]{ }^{\#} \hat{\boldsymbol{R}}^{T} \\
& -\tilde{\boldsymbol{K}}_{\# \hat{\boldsymbol{R}} \hat{\boldsymbol{G}}^{T}}\left[\check{\boldsymbol{R}} \boldsymbol{m}_{G}\right]+{ }^{\#} \hat{\boldsymbol{R}} \hat{\boldsymbol{G}}^{T} \check{\boldsymbol{R}}\left[\operatorname{spin}\left(\boldsymbol{m}_{G}\right) \check{\boldsymbol{G}}^{\#} \check{\boldsymbol{R}}^{T} \hat{\boldsymbol{P}}\right. \\
& \left.+\left(\overline{\boldsymbol{Q}}^{T}-\boldsymbol{m}_{G}^{\mathrm{f}} \otimes \boldsymbol{b}\right) \boldsymbol{B} \check{\boldsymbol{P}}^{\#} \check{\boldsymbol{R}}^{T} \hat{\boldsymbol{P}}-\boldsymbol{M}_{G}^{\mathrm{d}}\left(\check{\boldsymbol{R}}^{T} \hat{\boldsymbol{G}}+\check{\boldsymbol{G}}^{\#} \check{\boldsymbol{R}}^{T} \hat{\boldsymbol{P}}\right)\right]^{\#} \hat{\boldsymbol{R}}^{T} .
\end{aligned}
$$

Adding (103), (107), (114), and (119), it turns out that

$$
\Delta\left(\overline{\boldsymbol{q}}^{\mathrm{i}}+\overline{\boldsymbol{q}}^{\mathrm{l}}\right) \cdot \delta \overline{\boldsymbol{a}}+\Delta \hat{\boldsymbol{q}}^{1} \cdot \delta \hat{\boldsymbol{a}}+\Delta \tilde{\boldsymbol{q}}^{1} \cdot \delta \tilde{\boldsymbol{a}}=\overline{\boldsymbol{K}} \Delta \overline{\boldsymbol{a}} \cdot \delta \overline{\boldsymbol{a}}+\hat{\boldsymbol{K}}_{1} \Delta \hat{\boldsymbol{a}} \cdot \delta \hat{\boldsymbol{a}}+\tilde{\boldsymbol{K}}_{1} \Delta \tilde{\boldsymbol{a}} \cdot \delta \tilde{\boldsymbol{a}},
$$

where

$$
\overline{\boldsymbol{K}}=\overline{\boldsymbol{K}}_{\overline{\boldsymbol{q}}^{\mathrm{i}}}+\overline{\boldsymbol{K}}_{\overline{\boldsymbol{q}}^{1}}, \quad \hat{\boldsymbol{K}}_{1}=\hat{\boldsymbol{K}}_{\overline{\boldsymbol{q}}^{1}}+\hat{\boldsymbol{K}}_{\hat{\boldsymbol{q}}^{1}}, \quad \tilde{\boldsymbol{K}}_{1}=\tilde{\boldsymbol{K}}_{\overline{\boldsymbol{q}}^{1}}+\tilde{\boldsymbol{K}}_{\hat{\boldsymbol{q}}^{1}}+\tilde{\boldsymbol{K}}_{\tilde{\boldsymbol{q}}^{1}}
$$

4.5. Contribution from the terms involving $\Delta \delta \overline{\boldsymbol{a}}, \Delta \delta \hat{\boldsymbol{a}}$, and $\Delta \delta \tilde{\boldsymbol{a}}$

From (70), (67), (50), and (35), recalling that $\Delta \delta \boldsymbol{a}=0$, it respectively turns out that

$$
\begin{aligned}
& \boldsymbol{v} \cdot \Delta \delta \overline{\boldsymbol{a}}=\check{\boldsymbol{K}}_{\boldsymbol{B}^{T}}[\boldsymbol{v}] \Delta \check{\boldsymbol{a}} \cdot \delta \check{\boldsymbol{a}}+\boldsymbol{B}^{T} \boldsymbol{v} \cdot \Delta \delta \check{\boldsymbol{a}},
\end{aligned}
$$

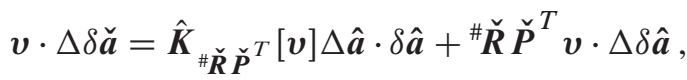

$$
\begin{aligned}
& \boldsymbol{v} \cdot \Delta \delta \hat{\boldsymbol{a}}=\tilde{\boldsymbol{K}}_{\# \hat{\boldsymbol{R}} \hat{\boldsymbol{P}}^{T}}[\boldsymbol{v}] \Delta \tilde{\boldsymbol{a}} \cdot \delta \tilde{\boldsymbol{a}}+{ }^{\#} \hat{\boldsymbol{R}} \hat{\boldsymbol{P}}^{T} \boldsymbol{v} \cdot \Delta \delta \tilde{\boldsymbol{a}}, \\
& \boldsymbol{v} \cdot \Delta \delta \tilde{\boldsymbol{a}}=\boldsymbol{K}_{\boldsymbol{M}^{T}}[\boldsymbol{v}] \Delta \boldsymbol{a} \cdot \delta \boldsymbol{a},
\end{aligned}
$$

where

$$
\begin{aligned}
\check{\boldsymbol{K}}_{\boldsymbol{B}^{T}}[\boldsymbol{v}] \Delta \check{\boldsymbol{a}} & =\left.\Delta\left(\boldsymbol{B}^{T} \boldsymbol{v}\right)\right|_{\boldsymbol{v}=\text { const }}, \\
\hat{\boldsymbol{K}}_{\# \check{\boldsymbol{\boldsymbol { P }}} \check{\check{\boldsymbol{P}}}}[\boldsymbol{v}] \Delta \hat{\boldsymbol{a}} & =\left.\Delta\left({ }^{\#} \check{\boldsymbol{R}}^{T} \boldsymbol{v}\right)\right|_{\boldsymbol{v}=\text { const }}, \\
\tilde{\boldsymbol{K}}_{\# \hat{\boldsymbol{R}} \hat{\boldsymbol{P}}^{T}}[\boldsymbol{v}] \Delta \tilde{\boldsymbol{a}} & =\left.\Delta\left({ }^{\#} \hat{\boldsymbol{R}} \hat{\boldsymbol{P}}^{T} \boldsymbol{v}\right)\right|_{\boldsymbol{v}=\mathrm{const}}, \\
\boldsymbol{K}_{\boldsymbol{M}^{T}}[\boldsymbol{v}] \Delta \boldsymbol{a} & =\left.\Delta\left(\boldsymbol{M}^{T} \boldsymbol{v}\right)\right|_{\boldsymbol{v}=\mathrm{const}} .
\end{aligned}
$$

The tensors $\check{\boldsymbol{K}}_{\boldsymbol{B}^{T}}[\boldsymbol{v}]$ and $\boldsymbol{K}_{\boldsymbol{M}^{T}}[\boldsymbol{v}]$ represent moment correction geometric stiffnesses. Their expressions are well known in the literature (e.g., [10,31,34]) and are reported in Appendix B for completeness. The tensors $\hat{\boldsymbol{K}}_{\# \check{\boldsymbol{R}} \check{\boldsymbol{P}}^{T}}[\boldsymbol{v}]$ and $\tilde{\boldsymbol{K}}_{\# \hat{\boldsymbol{R}} \hat{\boldsymbol{P}}^{T}}[\boldsymbol{v}]$ comprise two contributions: an equilibrium projection geometric stiffness, related to the linearization of the projectors $\check{\boldsymbol{P}}$ and $\hat{\boldsymbol{P}}$, respectively, and a rotational geometric stiffness, related to the linearization of the rotation tensors $\check{\boldsymbol{R}}$ and $\hat{\boldsymbol{R}}$, respectively. Exploiting (85) and their check counterparts, (55), (67), (41), and (50), it turns out that

$$
\begin{aligned}
& \hat{\boldsymbol{K}}_{\# \check{\boldsymbol{R}} \check{\boldsymbol{P}}} \check{\boldsymbol{v}}^{T}[\boldsymbol{v}]=-{ }^{\#} \check{\boldsymbol{R}}\left[\operatorname{Spin}\left(\check{\boldsymbol{P}}^{T} \boldsymbol{v}\right) \check{\boldsymbol{G}}+\check{\boldsymbol{G}}^{T}\left[\operatorname{Spin}\left(\check{\boldsymbol{P}}^{T} \boldsymbol{v}\right)\right]^{T}{ }^{\mathrm{I}} \boldsymbol{I} \check{\boldsymbol{P}}^{\#} \check{\boldsymbol{R}}^{T}\right. \\
& \tilde{\boldsymbol{K}}_{\# \hat{\boldsymbol{R}} \hat{\boldsymbol{P}}^{T}}[\boldsymbol{v}]={ }^{\#} \hat{\boldsymbol{R}}\left[\operatorname{Spin}\left(\hat{\boldsymbol{P}}^{T} \boldsymbol{v}\right) \hat{\boldsymbol{G}}+\hat{\boldsymbol{G}}^{T}\left[\operatorname{Spin}\left(\hat{\boldsymbol{P}}^{T} \boldsymbol{v}\right)\right]^{T}{ }^{\mathrm{b}} \boldsymbol{I} \hat{\boldsymbol{P}}\right]{ }^{\#} \hat{\boldsymbol{R}}^{T},
\end{aligned}
$$

where the classical construct can be recognized (e.g., [7,31]). 


\subsection{Consistent tangent stiffness tensor}

By (102), (121), (123), (98), (70), (67), (50), and (35), the following algorithm for the computation of the consistent tangent stiffness tensor $\boldsymbol{K}$ is obtained:

$$
\begin{aligned}
& \Delta(\boldsymbol{q} \cdot \delta \boldsymbol{a})= \\
& \overline{\boldsymbol{K}} \Delta \overline{\boldsymbol{a}} \cdot \delta \overline{\boldsymbol{a}}+\hat{\boldsymbol{K}}_{1} \Delta \hat{\boldsymbol{a}} \cdot \delta \hat{\boldsymbol{a}}+\tilde{\boldsymbol{K}}_{1} \Delta \tilde{\boldsymbol{a}} \cdot \delta \tilde{\boldsymbol{a}}+\overline{\boldsymbol{q}} \cdot \Delta \delta \overline{\boldsymbol{a}}+\hat{\boldsymbol{q}}^{1} \cdot \Delta \delta \hat{\boldsymbol{a}}+\tilde{\boldsymbol{q}}^{1} \cdot \Delta \delta \tilde{\boldsymbol{a}} \stackrel{\downarrow}{=} \delta \overline{\boldsymbol{a}}=\boldsymbol{B} \delta \check{\boldsymbol{a}}
\end{aligned}
$$

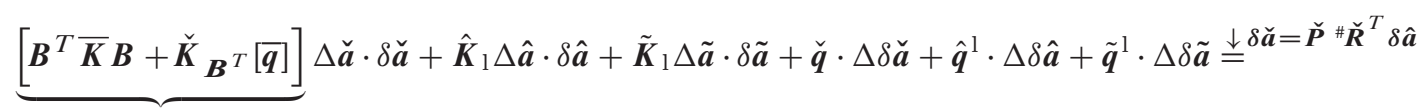

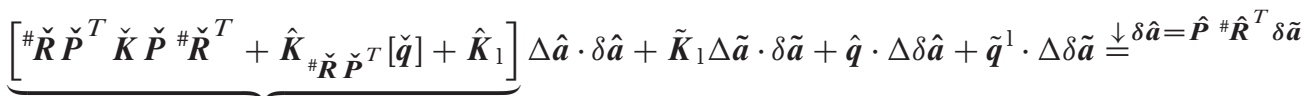

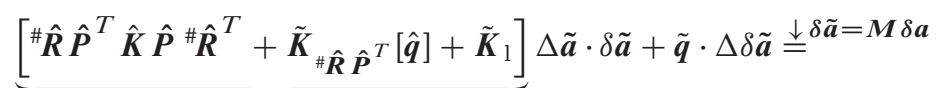

$$
\begin{aligned}
& \underbrace{\left[\boldsymbol{M}^{T} \tilde{\boldsymbol{K}} \boldsymbol{M}+\boldsymbol{K}_{\left.\boldsymbol{M}^{T}[\tilde{\boldsymbol{q}}]\right]}\right.} \Delta \boldsymbol{a} \cdot \delta \boldsymbol{a}= \\
& \boldsymbol{K} \Delta \boldsymbol{a} \cdot \delta \boldsymbol{a},
\end{aligned}
$$

where the tensor $\overline{\boldsymbol{K}}$ is defined in (122), and the tensors $\check{\boldsymbol{K}}, \hat{\boldsymbol{K}}, \tilde{\boldsymbol{K}}$, and $\boldsymbol{K}$, defined by the underbraces, are given by

$$
\begin{aligned}
& \check{\boldsymbol{K}}=\boldsymbol{B}^{T} \overline{\boldsymbol{K}} \boldsymbol{B}+\check{\boldsymbol{K}}_{\boldsymbol{B}^{T}}[\overline{\boldsymbol{q}}],
\end{aligned}
$$

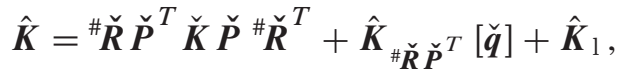

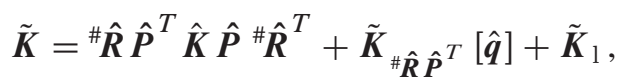

$$
\begin{aligned}
& \boldsymbol{K}=\boldsymbol{M}^{T} \tilde{\boldsymbol{K}} \boldsymbol{M}+\boldsymbol{K}_{\boldsymbol{M}^{T}}[\tilde{\boldsymbol{q}}] .
\end{aligned}
$$

When no distributed loads are present, the algorithms (127) and (128) reduce to the classical construct (e.g., [7, 10,31]), except that two filtering steps appear here instead of one.

Approximate contributions to the tangent stiffness tensor due to distributed follower loads were provided in [24]. Of course, according to that approach, only the terms arising from the linearization of (100) would appear.

\subsection{Small strain assumption}

Under small strain assumption, the following approximations are enforced:

$$
\overline{\boldsymbol{E}} \approx \overline{\boldsymbol{\varepsilon}}=\operatorname{sym} \nabla_{p} \overline{\boldsymbol{u}}=\operatorname{sym}(\overline{\boldsymbol{F}}-\boldsymbol{I}), \quad p+\overline{\boldsymbol{u}}(p)-G \approx p-G, \quad A^{\prime} \approx A .
$$

The expressions of $\boldsymbol{l}, \boldsymbol{f}, \boldsymbol{m}_{G}, \overline{\boldsymbol{q}}^{1}, \boldsymbol{M}_{G}^{\mathrm{d}}$ introduced earlier get simplified accordingly. As a consequence, some contributions to the consistent tangent stiffness tensor drop. In particular, the terms involving $\overline{\boldsymbol{Q}}$ in (112), (115), and (120), as well as the terms involving $\boldsymbol{b}$ in (104), (108), (112), (115), (116), and (120), disappear. Moreover, the core-element equilibrium weak formulation, that is, the last equality in (88), reduces to a geometrically linear equilibrium formulation, thus enabling the use of existing high-performance linear elements for the computation of the material tangent stiffness tensor $\overline{\boldsymbol{K}}_{\overline{\boldsymbol{q}}^{\mathrm{i}}}$.

\section{NUMERICAL RESULTS}

The proposed corotational framework has been validated by means of an extensive numerical campaign. Several geometrically nonlinear benchmark problems (e.g., [25]) have been considered and analyzed by means of the MATLAB toolkit described in Appendix A, using as core element a combination of the DKT plate $[14,27,28]$ and of the OPT membrane [29]. The obtained numerical results are reported in the following. 


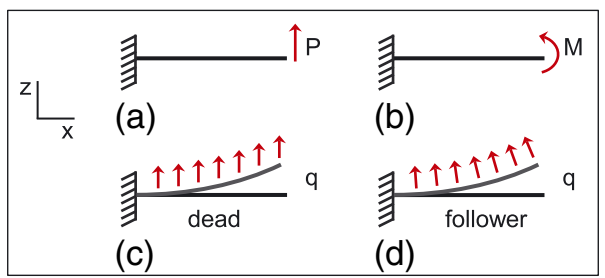

Figure 3. Cantilever load conditions: (a) end shear force, (b) end bending moment, (c) transverse distributed dead load, (d) transverse distributed follower load.

Three different structured meshes, that is, a coarse one, an intermediate one, and a fine one, were considered for each problem. In the nonlinear solution procedure, the full Newton-Raphson method was used. The simultaneous $0.5 \%$ force tolerance and $1 \%$ displacement tolerance were employed as convergence criterion [25]. The results did not significantly change when tighter tolerances were enforced. The automatic load incrementation scheme described in [25] was adopted. Throughout the scheme, the maximum load was automatically subdivided into NINC load increments that were not necessarily uniform. At the end of each load increment, a converged intermediate solution was obtained. The total number of iterations required to obtain the NINC converged intermediate solutions is denoted by NITER. Both NINC and NITER are reported in Tables I-VIII, revealing some computational saving with respect to the standard formulation adopted in [25]. Additional data points, obtained with a uniform load incrementation scheme, were employed to enhance the quality of the load-deflection curves.

\subsection{Clamped cantilever}

A clamped cantilever with rectangular cross section, width $b=1$, thickness $h=0.1$, Young's modulus $E=1.2 \times 10^{6}$, and zero Poisson's ratio, is subjected to four load conditions (Figure 3). The tip $x$-deflection and $z$-deflection, $u_{\text {tip }}$ and $w_{\text {tip }}$, respectively, are reported in Table I.

5.1.1. Cantilever subjected to end shear force. The cantilever length is $L=10$, and the maximum end shear force is $P^{\max }=4 P_{0}$, with $P_{0}=E I / L^{2}=1$ and $I$ the second moment of area. Figure 4(a) shows the deformation history obtained with the depicted $16 \times 1$ mesh. The relevant load-deflection curves are plotted in Figure 4(b), where the theoretical solution in [26] is reported for comparison.

Table I. Clamped cantilever subjected to the four load conditions depicted in Figure 3.

\begin{tabular}{llcccc}
\hline Load condition & Mesh & $12 \times 1$ & $16 \times 1$ & $24 \times 1$ & Reference [26] \\
\hline End shear force & $-u_{\text {tip }}$ & 3.290 & 3.290 & 3.290 & 3.289 \\
& $w_{\text {tip }}$ & 6.707 & 6.705 & 6.703 & 6.700 \\
& NINC & 4 & 7 & 4 & - \\
& NITER & 26 & 35 & 28 & - \\
End bending moment & $-u_{\text {tip }}$ & 12.000 & 12.000 & 12.000 & 12.000 \\
& $w_{\text {tip }}$ & 0.011 & 0.001 & 0.000 & 0.000 \\
& NINC & 19 & 24 & 23 & - \\
Distributed dead load & NITER & 149 & 163 & 159 & - \\
& $-u_{\text {tip }}$ & 7.177 & 7.173 & 7.169 & 7.165 \\
& $w_{\text {tip }}$ & 8.971 & 8.963 & 8.956 & 8.946 \\
& NINC & 11 & 8 & 8 & - \\
Nistributed follower load & NITER & 57 & 49 & 44 & - \\
& $-u_{\text {tip }}$ & 13.898 & 13.890 & 13.884 & 13.882 \\
& $w_{\text {tip }}$ & 1.546 & 1.524 & 1.510 & 1.500 \\
& NINC & 11 & 11 & 12 & - \\
\hline
\end{tabular}




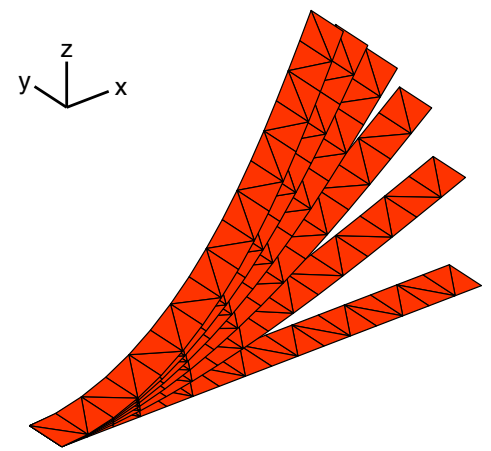

(a)

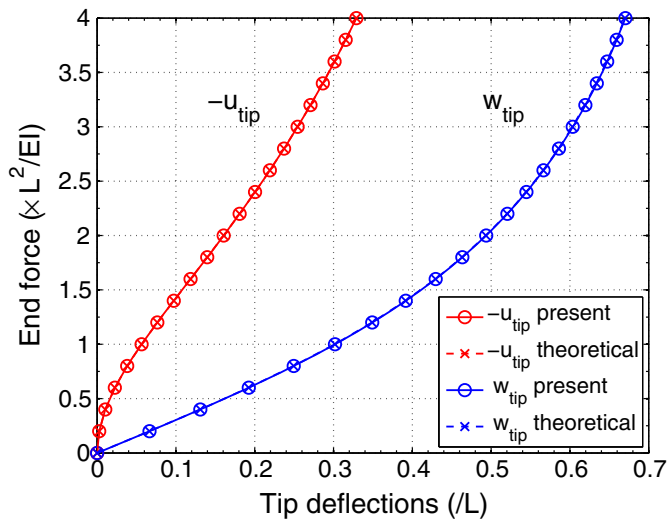

(b)

Figure 4. Cantilever subjected to end shear force. (a) Reference and deformed configurations at five uniformly spaced load levels and (b) load-deflection curves.

5.1.2. Cantilever subjected to end bending moment. The cantilever length is $L=12$. The deformed configuration is a circular arc with radius $R=E I / M$. The analytical deflections are

$$
u_{\text {tip }}=\left(\frac{\sin \left(M / M_{0}\right)}{M / M_{0}}-1\right) L, \quad w_{\text {tip }}=\frac{1-\cos \left(M / M_{0}\right)}{M / M_{0}} L
$$

with $M_{0}=E I / L$. The maximum end moment $M^{\max }$ is taken to be $2 \pi M_{0}$, at which the beam will be bent into a circle. Figure 5(a) shows the deformation history obtained with the depicted $16 \times 1$ mesh. The relevant load-deflection curves are plotted in Figure 5(b), where the analytical solution is reported for comparison.

5.1.3. Cantilever subjected to transverse uniformly distributed load. The cantilever length is $L=$ 10 , and the maximum transverse distributed dead (respectively, follower) load is $q^{\max }=40 q_{0}$, with $q_{0}=E I / L^{3}$. Figure 6(a) (respectively, Figure 7(a)) shows the deformation history obtained with the depicted 16×1 mesh. The relevant load-deflection curves are plotted in Figure 6(b) (respectively, Figure 7(b)), where the reference solution obtained by [26] is reported for comparison.

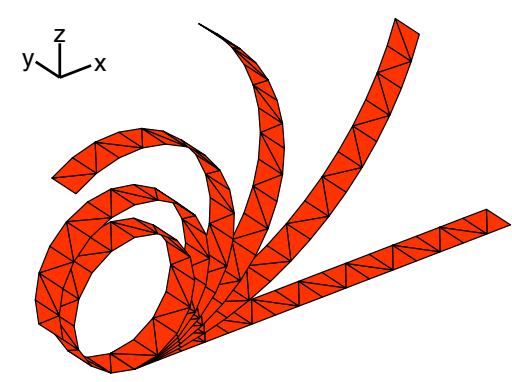

(a)

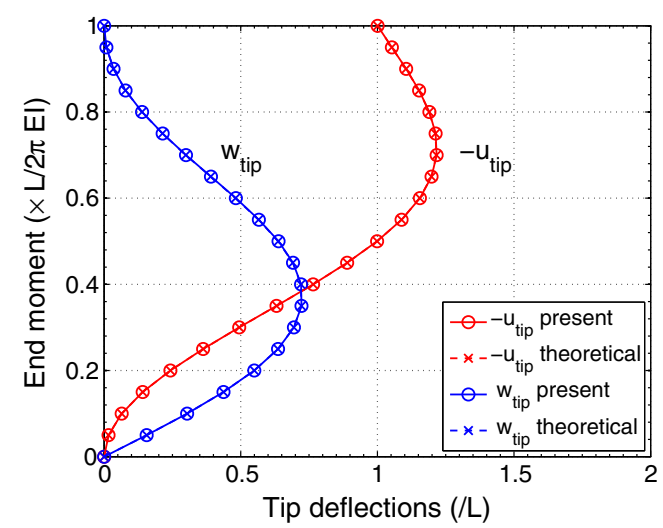

(b)

Figure 5. Cantilever subjected to end bending moment. (a) Reference and deformed configurations at five uniformly spaced load levels and (b) load-deflection curves. 


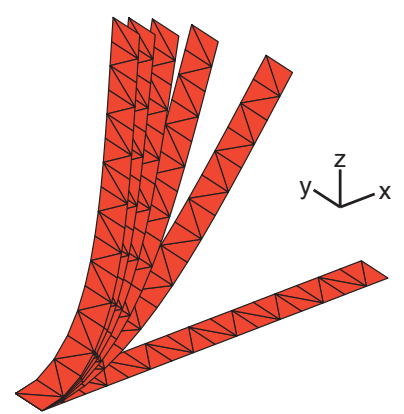

(a)

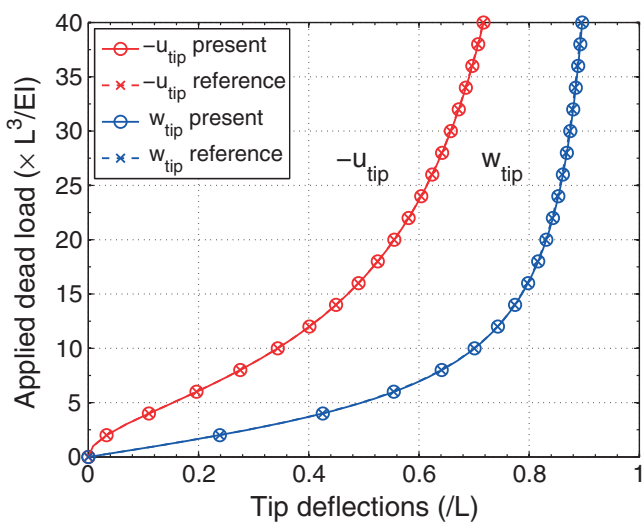

(b)

Figure 6. Cantilever subjected to transverse uniformly distributed dead load. (a) Reference and deformed configurations at five uniformly spaced load levels and (b) load-deflection curves.

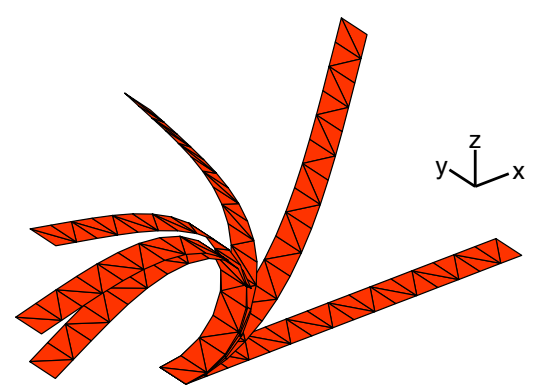

(a)

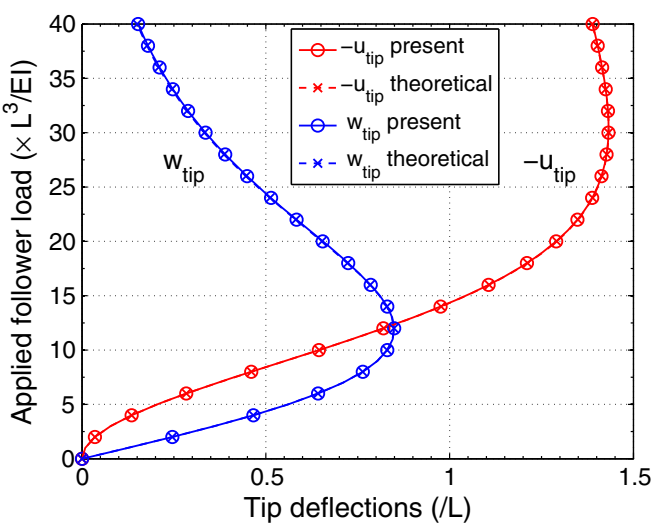

(b)

Figure 7. Cantilever subjected to transverse uniformly distributed follower load. (a) Reference and deformed configurations at five uniformly spaced load levels and (b) load-deflection curves.

\subsection{Clamped square plate under transverse uniformly distributed dead load}

A square plate of side $L=100$ and thickness $h=1$, clamped along all four boundaries, is subjected to transverse uniformly distributed dead load $q$. Young's modulus is $E=2.1 \times 10^{6}$, and Poisson's ratio is $v=0.316$. The maximum load is $q_{\max }=20$. Owing to symmetry, one-quarter of the plate is modeled. Table II reports the $z$-deflection $w_{C}$ at the plate central point $C$ under maximum load. Figure 8 portraits the load level against $w_{C}$ obtained with the $64 \times 64$ mesh. The analytical solution is plotted for comparison. It is given by [35]: $w_{C}=-2.5223 w_{0}$, where $w_{0}$ solves:

$$
\left(\frac{w_{0}}{h}\right)^{3}+0.2552 \frac{w_{0}}{h}=0.00013333 \frac{q L^{4}}{D h},
$$

with $D$ being the plate flexural stiffness.

\subsection{Slit annular plate subjected to lifting line force}

A slit annular plate of inner radius $R_{\mathrm{i}}=6$, outer radius $R_{\mathrm{O}}=10$, and thickness $h=0.03$ is considered. Young's modulus is $E=21 \times 10^{6}$, and Poisson's ratio vanishes. A line force $P$ is applied at one end of the slit, whereas the other end of the slit is fully clamped. The maximum load level is $P^{\max }=0.8$. Table III reports the $z$-deflections $w_{A}$ and $w_{B}$ at the inner and outer 
Table II. Clamped square plate subjected to dead distributed normal load.

\begin{tabular}{llllc}
\hline Mesh & $16 \times 16$ & $32 \times 32$ & $64 \times 64$ & Reference [35] \\
\hline$-w_{C}$ & 2.618 & 2.612 & 2.610 & 2.612 \\
NINC & 1 & 1 & 1 & - \\
NITER & 9 & 9 & 9 & - \\
\hline
\end{tabular}

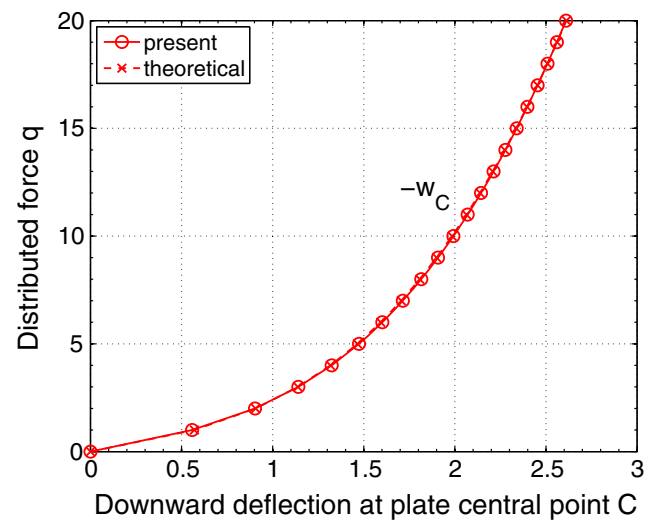

Figure 8. Clamped square plate subjected to dead distributed normal load. Load-deflection curve.

Table III. Slit annular plate subjected to lifting line force.

\begin{tabular}{lllll}
\hline Mesh & $6 \times 30$ & $8 \times 60$ & $10 \times 80$ & Reference [25] \\
\hline$w_{A}$ & 13.402 & 13.776 & 13.818 & 13.891 \\
$w_{B}$ & 17.017 & 17.411 & 17.455 & 17.528 \\
NINC & 37 & 23 & 23 & 67 \\
NITER & 219 & 163 & 157 & 346 \\
\hline
\end{tabular}

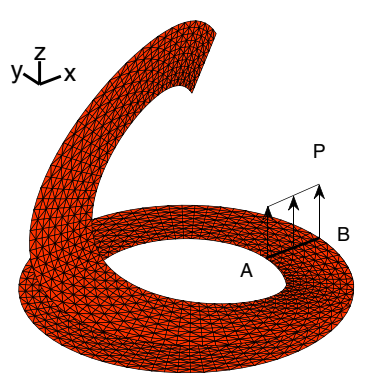

(a)

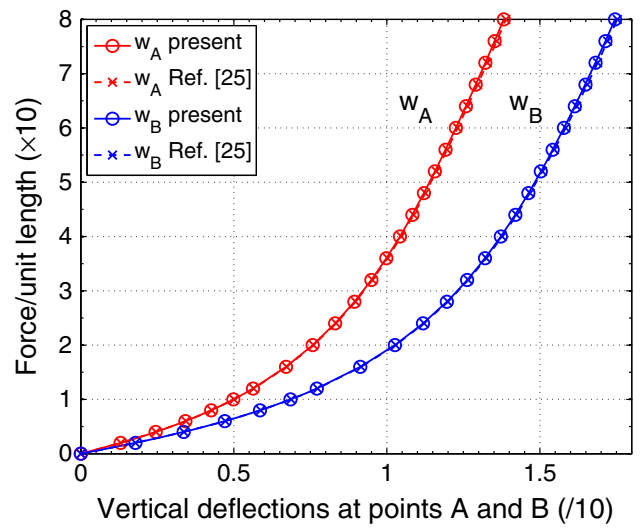

(b)

Figure 9. Slit annular plate subjected to lifting line force. (a) Reference and final configurations and (b) load-deflection curves.

slit extremal points, $A$ and $B$, respectively. Figure 9(a) shows the reference and deformed configurations, obtained with the $10 \times 80$ mesh. Load-deflection curves are depicted in Figure 9(b). The results in [25] are plotted for comparison. 


\subsection{Hemispherical shell subjected to alternating radial forces}

A hemispherical shell of radius $R=10$, thickness $h=0.04$, and with an $18^{\circ}$ circular cutout at its pole is loaded by alternating radial point forces $P$ 's at $90^{\circ}$ intervals (Figure 10(a)). Young's modulus is $E=6.825 \times 10^{7}$, and Poisson's ratio is $v=0.3$. The maximum load level is $P^{\max }=400$. Owing to symmetry, one-quarter of the shell is modeled. Table IV reports the radial displacements $u_{A}$ and $v_{B}$ at points $A$ and $B$, respectively. Figure 10(b) shows the deformed configuration obtained with the $64 \times 64$ mesh. Load-deflection curves are depicted in Figure 11. The results in [25] are plotted for comparison.

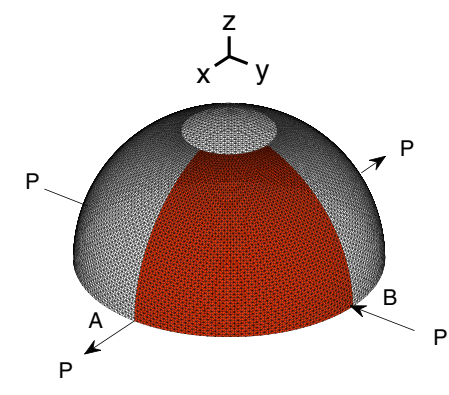

(a)

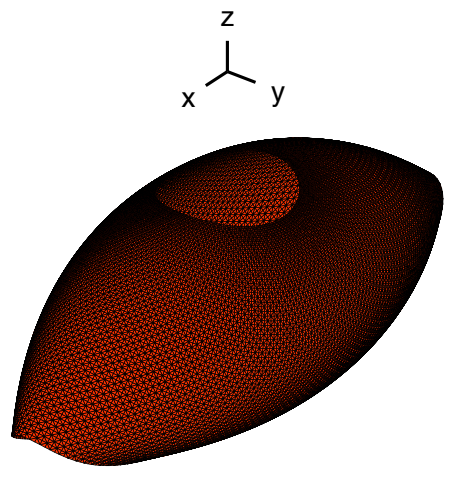

(b)

Figure 10. Hemispherical shell subjected to alternating radial forces. (a) Reference and (b) final configurations.

Table IV. Hemispherical shell subjected to alternating radial forces.

\begin{tabular}{lcccc}
\hline Mesh & $16 \times 16$ & $32 \times 32$ & $64 \times 64$ & Reference [25] \\
\hline$u_{A}$ & 3.855 & 4.043 & 4.071 & 4.067 \\
$-v_{B}$ & 7.467 & 8.057 & 8.142 & 8.178 \\
NINC & 12 & 13 & 13 & 27 \\
NITER & 86 & 87 & 87 & 136 \\
\hline
\end{tabular}

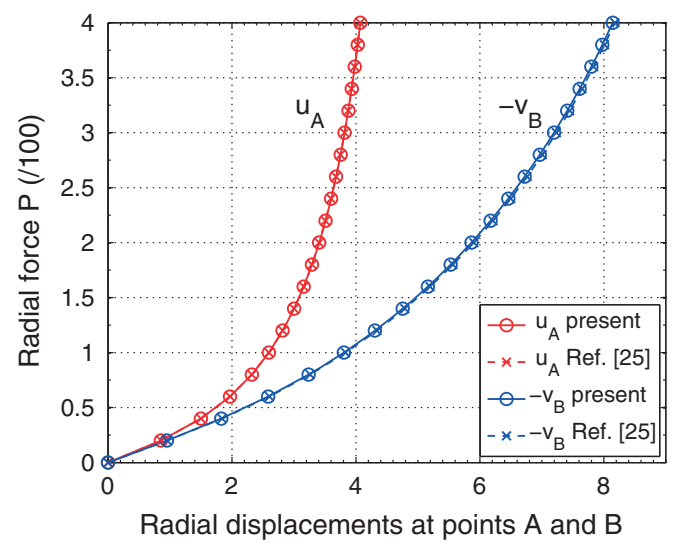

Figure 11. Hemispherical shell subjected to alternating radial forces. Load-deflection curves. 


\subsection{Pullout of an open-ended cylindrical shell}

An open-ended cylinder of radius $R=4.953$, length $L=10.35$, and thickness $h=0.094$ is pulled by a pair of radial forces $P$ 's (Figure 12(a)). Young's modulus is $E=10.5 \times 10^{6}$, and Poisson's ratio is $v=0.3125$. The maximum load level is $P^{\max }=40,000$. Owing to symmetry, one-eighth of the shell is modeled. Table $\mathrm{V}$ reports the radial deflections $w_{A}, u_{B}$, and $u_{C}$ at points $A, B$, and $C$, respectively. Figure 12(b) shows the deformed configuration obtained with the $24 \times 36$ mesh. Load-deflection curves are depicted in Figure 13. The results in [25] are plotted for comparison.

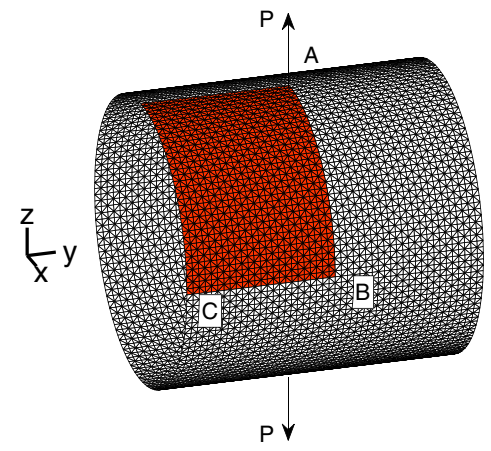

(a)

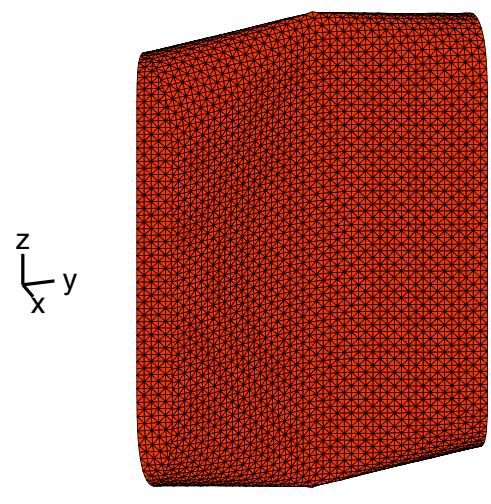

(b)

Figure 12. Pulled open-ended cylindrical shell. (a) Reference and (b) final configurations.

Table V. Pulled open-ended cylindrical shell.

\begin{tabular}{lcccc}
\hline Mesh & $12 \times 18$ & $16 \times 24$ & $24 \times 36$ & Reference [25] \\
\hline$w_{A}$ & 2.753 & 2.748 & 2.745 & 2.768 \\
$-u_{B}$ & 4.562 & 4.555 & 4.550 & 4.551 \\
$-u_{C}$ & 3.258 & 3.269 & 3.278 & 3.269 \\
NINC & 21 & 20 & 11 & 18 \\
NITER & 113 & 119 & 80 & 94 \\
\hline
\end{tabular}

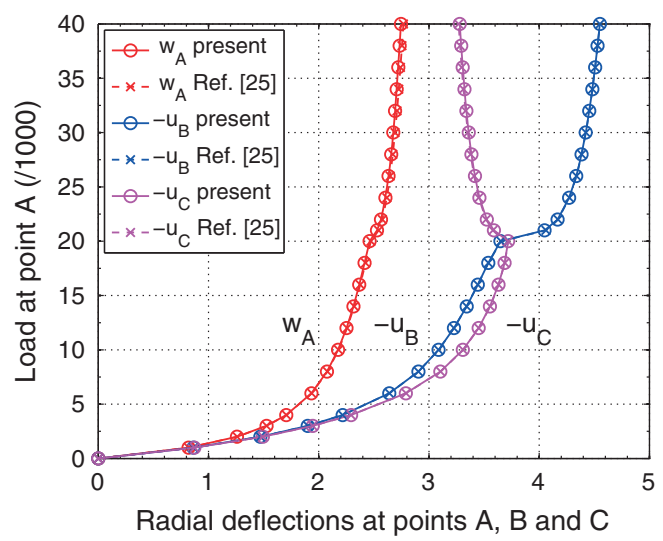

Figure 13. Pulled open-ended cylindrical shell. Load-deflection curves. 


\subsection{Pinched cylindrical shell mounted over rigid diaphragms}

A cylindrical shell of radius $R=100$, length $L=200$, and thickness $h=1$ is mounted on rigid end diaphragms over which the $x$-displacement and $z$-displacement, $u$ and $w$, respectively, are restrained. The cylindrical shell is pinched by a pair of opposite forces $P$ 's (Figure 14(a)). Young's modulus is $E=30 \times 10^{3}$, and Poisson's ratio is $v=0.3$. The maximum load level is $P^{\max }=12,000$. Owing to symmetry, one-eighth of the shell is modeled. Table VI reports the radial deflections $w_{A}$ and $u_{B}$ at points $A$ and $B$, respectively. Figure 14(b) shows the deformed configuration obtained with the $48 \times 48$ mesh. Load-deflection curves are depicted in Figure 15. The results in [25] are plotted for comparison. Figure 7(b) of [25] shows that solutions yielded by two successively refined meshes (i.e., $40 \times 40$ and $48 \times 48$ ) are slightly different: The finer one has been used here for comparison.

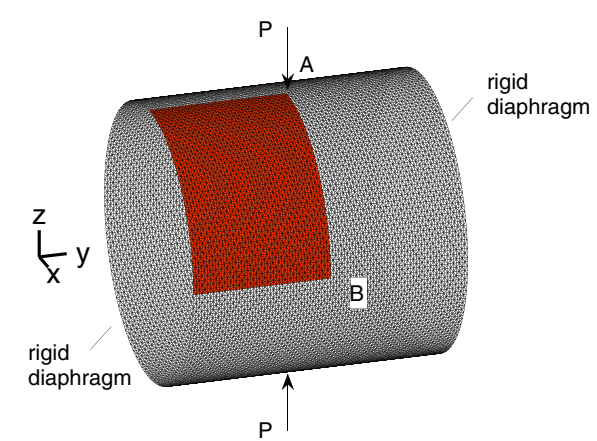

(a)

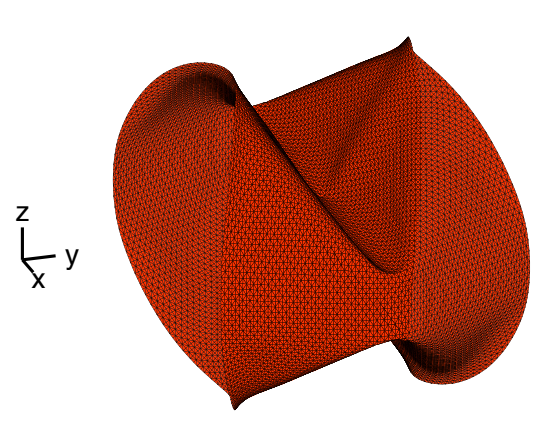

(b)

Figure 14. Pinched cylinder mounted over rigid end diaphragms. (a) Reference and (b) final configurations.

Table VI. Pinched cylinder mounted over rigid end diaphragms.

\begin{tabular}{lllll}
\hline Mesh & $40 \times 40$ & $48 \times 48$ & $64 \times 64$ & Reference [25] \\
\hline$-w_{A}$ & 84.010 & 83.900 & 83.909 & 83.102 \\
$u_{B}$ & 35.739 & 35.560 & 35.593 & 34.272 \\
NINC & 48 & 47 & 44 & 70 \\
NITER & 341 & 313 & 293 & 406 \\
\hline
\end{tabular}

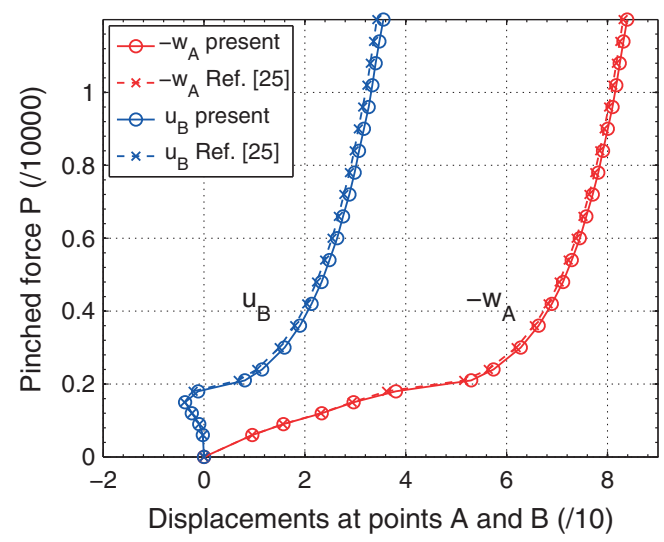

Figure 15. Pinched cylinder mounted over rigid end diaphragms. Load-deflection curves. 


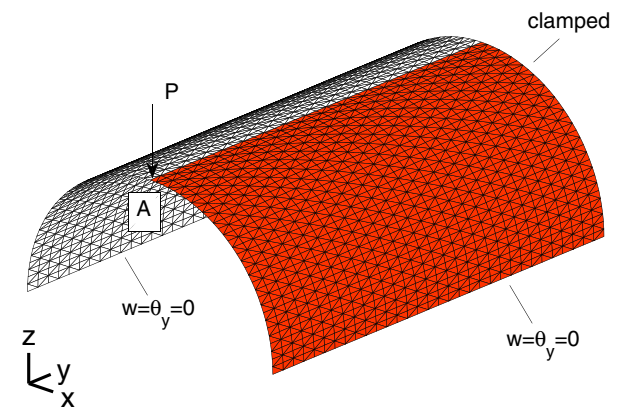

(a)

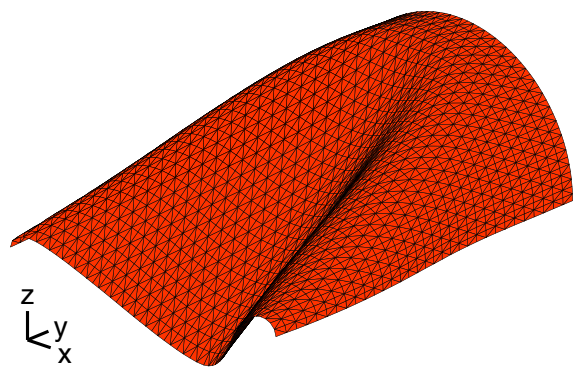

(b)

Figure 16. Pinched semi-cylindrical shell. (a) Reference and (b) final configurations.

Table VII. Pinched semi-cylindrical shell.

\begin{tabular}{lllll}
\hline Mesh & $24 \times 24$ & $32 \times 32$ & $40 \times 40$ & Reference [25] \\
\hline$-w_{A}$ & 1.704 & 1.706 & 1.707 & 1.715 \\
NINC & 12 & 13 & 13 & 28 \\
NITER & 93 & 97 & 97 & 136 \\
\hline
\end{tabular}

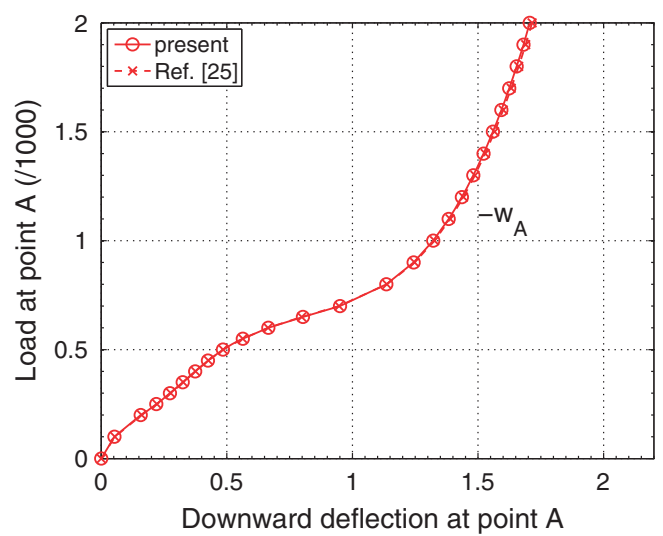

Figure 17. Pinched semi-cylindrical shell. Load-deflection curve.

\subsection{Pinched semi-cylindrical shell}

A semi-cylindrical shell of radius $R=1.016$, length $L=3.048$, and thickness $h=0.03$ is subjected to an end pinching force $P$ at the middle of the free-hanging circumferential periphery. The other circumferential periphery is fully clamped (Figure 16(a)). Along its longitudinal edges, the $z$-deflection $w$ and the rotation $\theta_{y}$ about the $y$-axis are restrained. Young's modulus is $E=2.0685 \times 10^{6}$, and Poisson's ratio is $v=0.3$. The maximum load level is $P^{\max }=2,000$. Owing to symmetry, one-half of the shell is modeled. Table VII reports the $z$-deflection $w_{A}$ at point $A$. Figure 16(b) shows the deformed configuration obtained with the $32 \times 32$ mesh. Load-deflection curves are depicted in Figure 17. The results in [25] are plotted for comparison.

\subsection{Hinged cylindrical roof subjected to a central pinching force}

A hinged semi-cylindrical roof of radius $R=2,540$, half-length $L=254$, half-aperture $\theta=0.1$ radian, and thickness $h=12.7$, is subjected to a central pinching force $P$ (Figure 18(a)). Along the hinged edges, all nodal translations are restrained. Young's modulus is $E=3,102.75$, and Poisson's ratio is $v=0.3$. The analysis is performed under displacement control, up to 


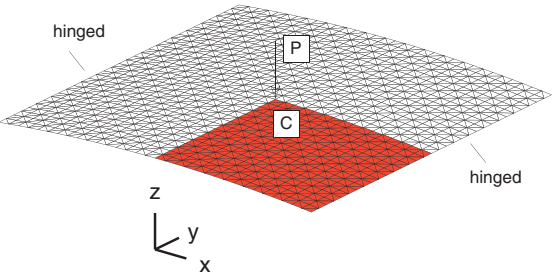

(a)

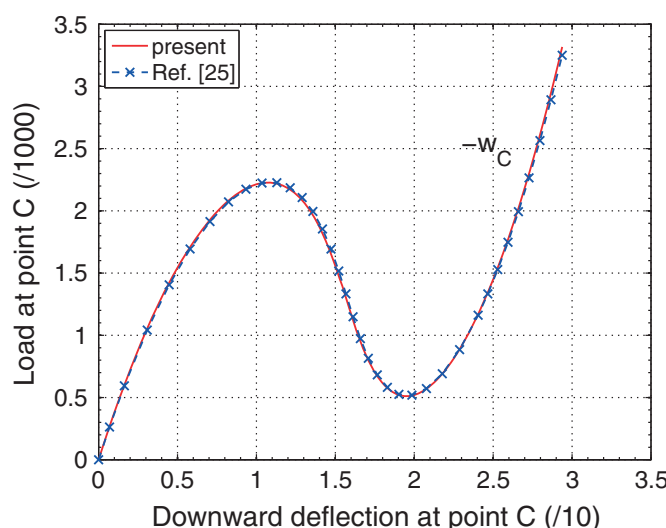

(b)

Figure 18. Hinged cylindrical roof subjected to a central pinching force. (a) Reference configuration and (b) load-deflection curve.

Table VIII. Hinged cylindrical roof subjected to a central pinching force.

\begin{tabular}{lrrrc}
\hline Mesh & $8 \times 8$ & $12 \times 12$ & $16 \times 16$ & Reference [25] \\
\hline$P$ & 3,283 & 3,308 & 3,317 & 3,250 \\
NINC & 1 & 1 & 1 & - \\
NITER & 8 & 7 & 7 & - \\
\hline
\end{tabular}

a $z$-displacement $w_{C}^{\max }=29.374$. Owing to symmetry, one-quarter of the roof is modeled. Table VIII reports the force $P$ applied at point $C$. Load-deflection curves obtained with the $16 \times 16$ mesh are depicted in Figure 18(b). The results in [25] are plotted for comparison.

\section{CONCLUSIONS}

A polar decomposition based corotational formulation for three-node triangular shell elements with six DOFs per node has been presented. Original formulas for the computation of rigid body motion, projector operator, nodal residual vector, and consistent tangent stiffness tensor have been derived. Distributed dead and follower loads have been rigorously taken into account for the first time in the context of a corotational kinematic description, showing that different contributions arise in the nodal residual vector and in the consistent tangent stiffness tensor. The present approach has been validated using several numerical benchmarks and turned out to compare favorably with standard Lagrangian formulations. Its simplicity and effectiveness make it attractive for many applications, involving, for example, contact mechanics or fluid-structure interaction.

\section{APPENDIX A: MATLAB TOOLKIT}

A MATLAB toolkit implementing the present formulation is provided as supplementary material for the interested reader. The toolkit can also be obtained from the authors. As pointed out in [10], 'the key operations of adding and removing rigid body motions can be visualized as a front end filter that lies between the assembler/solver and the element library'. Accordingly, the toolkit is composed of the following two main routines:

(1) filter_in, implementing the algorithm derived in Section 2. It is called by the assembler before the core-element routine and has the purpose of removing rigid body motions. In particular, it transforms the element nodal parameters $\boldsymbol{a}$ into their filtered counterpart $\overline{\boldsymbol{a}}$ and the distributed 
dead (respectively, follower) loads into their pulled-back counterparts $\boldsymbol{R}^{T} \boldsymbol{l}^{\mathrm{d}}$ (respectively, $l^{\mathrm{f}} A^{\prime} / A$ ), to be passed to the core element. Its dependencies are the routines

(i) rot2spin, transforming variations of nodal rotation vectors to nodal spins and returning the tensor $\boldsymbol{M}$ (Section 2.3.1);

(ii) hat, computing the rototranslation $(\hat{\boldsymbol{R}}, \boldsymbol{t})$ and the related tensors $\hat{\boldsymbol{G}}, \hat{\boldsymbol{P}}$ (Section 2.3.2);

(iii) chk, computing the rotation $\check{\boldsymbol{R}}$ and the related tensors $\check{\boldsymbol{G}}, \check{\boldsymbol{P}}$ (Section 2.3.3);

(iv) spin2rot, transforming filtered nodal spins to variations of filtered nodal rotation vectors and returning the tensor $\boldsymbol{B}$ (Section 2.3.4);

(2) filter_out, implementing the equations presented in Sections 3 and 4. It is called by the assembler after the core-element routine, and has the purpose of computing the nodal residual vector $\boldsymbol{q}$ and the consistent tangent stiffness tensor $\boldsymbol{K}$, respectively through the algorithms (97), (98), and (127), (128). Dependencies of filter_out are the routines

(i) D_Bt, returning $\check{\boldsymbol{K}}_{\boldsymbol{B}^{T}}[\boldsymbol{v}]$, Equation (B.3);

(ii) D_shp_R_Gt, returning $\hat{\boldsymbol{K}}_{\# \check{\boldsymbol{R}} \check{\boldsymbol{G}}^{T}}[\boldsymbol{v}]$, Equation (111), or $\tilde{\boldsymbol{K}}_{\# \hat{\boldsymbol{R}} \hat{\boldsymbol{G}}^{T}}[\boldsymbol{v}]$, Equation (118);

(iii) D_shp_R_Pt, returning $\hat{\boldsymbol{K}}_{\# \check{\boldsymbol{\boldsymbol { R }}} \check{\boldsymbol{P}}^{T}}[\boldsymbol{v}]$, Equation (125), or $\tilde{\boldsymbol{K}}_{\# \hat{\boldsymbol{R}} \hat{\boldsymbol{P}}^{T}}[\boldsymbol{v}]$, Equation (126);

(iv) D_Mt, returning $\boldsymbol{K}_{\boldsymbol{M}^{T}}[\boldsymbol{v}]$, equation (B.1).

Computations are performed using coordinates in the element reference frame $\{\boldsymbol{e}, \boldsymbol{h}, \boldsymbol{n}\}$, which is returned by the routine geometry. Other auxiliary self-explanatory routines are included.

For the sake of completeness, a small-strain core-element implementation is provided as an example. In particular, a combination of the DKT plate $[14,27,28]$ and of the OPT membrane [29] is implemented in the routine DKT_OPT_shell: Details are omitted here, because that implementation is standard. Of course, any other small-strain or finite-strain triangular shell element with the same node and DOF configuration can be used in the present corotational framework.

This toolkit can be easily integrated into general-purpose finite element programs. The MATLAB code turned out to be quite efficient; moreover, it may serve as a guide, for example, for Fortran or $\mathrm{C}++$ implementations.

\section{APPENDIX B: MOMENT CORRECTION TENSORS $\boldsymbol{K}_{\boldsymbol{M}^{T}}[\boldsymbol{v}]$ AND $\check{\boldsymbol{K}}_{\boldsymbol{B}^{T}}[\boldsymbol{v}]$}

Let $\boldsymbol{v}=\left\{\boldsymbol{f}_{1} ; \boldsymbol{m}_{1} ; \boldsymbol{f}_{2} ; \boldsymbol{m}_{2} ; \boldsymbol{f}_{3} ; \boldsymbol{m}_{3}\right\}$. The tensor $\boldsymbol{K}_{\boldsymbol{M}^{T}}[\boldsymbol{v}]$ has the structure

$$
\boldsymbol{K}_{\boldsymbol{M}^{T}}[\boldsymbol{v}]=\operatorname{diag}\left[\mathbf{0} ; \boldsymbol{K}_{\boldsymbol{M}_{1}^{T}}\left[\boldsymbol{m}_{1}\right] ; \mathbf{0} ; \boldsymbol{K}_{\boldsymbol{M}_{2}^{T}}\left[\boldsymbol{m}_{2}\right] ; \mathbf{0} ; \boldsymbol{K}_{\boldsymbol{M}_{3}^{T}}\left[\boldsymbol{m}_{3}\right]\right],
$$

where (the $i$ subscript is omitted where no confusion may arise)

$$
\begin{gathered}
\boldsymbol{K}_{\boldsymbol{M}_{i}^{T}}[\boldsymbol{m}]=\frac{(1-\cos \vartheta)}{\vartheta^{2}}[\operatorname{spin}(\boldsymbol{m})-\boldsymbol{m} \otimes \vartheta]+\frac{(\vartheta-\sin \vartheta)}{\vartheta^{3}}[\boldsymbol{m} \otimes \vartheta+\vartheta \otimes \boldsymbol{m}+(\vartheta \cdot \boldsymbol{m}) \boldsymbol{I}] \\
+\frac{(\vartheta \sin \vartheta+2 \cos \vartheta-2)}{\vartheta^{4}}[\operatorname{spin}(\boldsymbol{m})(\vartheta \otimes \vartheta)]+\frac{(3 \sin \vartheta-\vartheta \cos \vartheta-2 \vartheta)}{\vartheta^{5}}[(\vartheta \cdot \boldsymbol{m}) \vartheta \otimes \vartheta]
\end{gathered}
$$

This expression of $\boldsymbol{K}_{\boldsymbol{M}_{i}^{T}}[\boldsymbol{m}]$ has been obtained by recasting results given in [34,36]. In particular, only functions with removable singularities around $\vartheta=0$ appear in (B.2), thus enabling effective computation by series.

Analogously, the tensor $\check{\boldsymbol{K}}_{\boldsymbol{B}^{T}}[\boldsymbol{v}]$ has the structure:

$$
\check{\boldsymbol{K}}_{\boldsymbol{B}^{T}}[\boldsymbol{v}]=\operatorname{diag}\left[\mathbf{0} ; \check{\boldsymbol{K}}_{\boldsymbol{B}_{1}^{T}}\left[\boldsymbol{m}_{1}\right] ; \mathbf{0} ; \check{\boldsymbol{K}}_{\boldsymbol{B}_{2}^{T}}\left[\boldsymbol{m}_{2}\right] ; \mathbf{0} ; \check{\boldsymbol{K}}_{\boldsymbol{B}_{3}^{T}}\left[\boldsymbol{m}_{3}\right]\right],
$$

where (e.g., $[7,10,34,36])$

$$
\check{\boldsymbol{K}}_{\boldsymbol{B}_{i}^{T}}[\boldsymbol{m}]=\left\{\eta[(\overline{\boldsymbol{\vartheta}} \cdot \boldsymbol{m}) \boldsymbol{I}+\overline{\boldsymbol{\vartheta}} \otimes \boldsymbol{m}-2 \boldsymbol{m} \otimes \bar{\vartheta}]+\mu \operatorname{spin}(\overline{\boldsymbol{\vartheta}})^{2} \boldsymbol{m} \otimes \bar{\vartheta}-\frac{1}{2} \operatorname{spin}(\boldsymbol{m})\right\} \boldsymbol{B}_{i},
$$


and

$$
\mu=\frac{d \eta / d \bar{\vartheta}}{\bar{\vartheta}}=\frac{\bar{\vartheta}^{2}+4 \cos \bar{\vartheta}+\bar{\vartheta} \sin \bar{\vartheta}-4}{4 \bar{\vartheta}^{4} \sin ^{2}\left(\frac{\bar{\vartheta}}{2}\right)}
$$

\section{ACKNOWLEDGEMENTS}

The authors express their sincere gratitude to Professor Franco Maceri for valuable comments on this work. The financial support of PRIN 2010-11, project 'Advanced mechanical modeling of new materials and technologies for the solution of 2020 European challenges' CUP n. F11J12000210001, is gratefully acknowledged.

\section{REFERENCES}

1. Sze KY, Chan WK, Pian THH. An eight-node hybrid-stress solid-shell element for geometric non-linear analysis of elastic shells. International Journal for Numerical Methods in Engineering 2002; 55(7):853-878. DOI: 10.1002/nme.535.

2. Wempner G. Finite elements, finite rotations and small strains of flexible shells. International Journal of Solids and Structures 1969; 5(2):117-153. DOI: 10.1016/0020-7683(69)90025-0.

3. Belytschko T, Hsieh BJ. Non-linear transient finite element analysis with convected co-ordinates. International Journal for Numerical Methods in Engineering 1973; 7(3):255-271. DOI: 10.1002/nme.1620070304.

4. Argyris JH, Balmer H, Doltsinis JS, Dunne PC, Haase M, Kleiber M, Malejannakis GA, Mlejnek H-P, Müller M, Scharpf DW. Finite element method-the natural approach. Computer Methods in Applied Mechanics and Engineering 1979; 17-18(1):1-106. DOI: 10.1016/0045-7825(79)90083-5.

5. Rankin CC, Brogan FA. An element independent corotational procedure for the treatment of large rotations. Journal of Pressure Vessel Technology 1986; 108(2):165-174. DOI: 10.1115/1.3264765.

6. Rankin CC, Nour-Omid B. The use of projectors to improve finite element performance. Computers and Structures 1988; 30(1-2):257-267. DOI: 10.1016/0045-7949(88)90231-3.

7. Nour-Omid B, Rankin CC. Finite rotation analysis and consistent linearization using projectors. Computer Methods in Applied Mechanics and Engineering 1991; 93(3):353-384. DOI: 10.1016/0045-7825(91)90248-5.

8. Crisfield MA. A consistent co-rotational formulation for non-linear, three-dimensional, beam-elements. Computer Methods in Applied Mechanics and Engineering 1990; 81(2):131-150. DOI: 10.1016/0045-7825(90)90106-V.

9. Peng X, Crisfield MA. A consistent co-rotational formulation for shells using the constant stress/constant moment triangle. International Journal for Numerical Methods in Engineering 1992; 35(9):1829-1847. DOI: 10.1002/nme.1620350907.

10. Felippa CA, Haugen B. A unified formulation of small-strain corotational finite elements: I. Theory. Computer Methods in Applied Mechanics and Engineering 2005; 194(21-24):2285-2335. DOI: 10.1016/j.cma.2004.07.035.

11. Crisfield MA, Moita GF. A co-rotational formulation for 2-D continua including incompatible modes. International Journal for Numerical Methods in Engineering 1996; 39(15):2619-2633. DOI: 10.1002/(SICI)10970207(19960815)39:15<2619::AID-NME969>3.0.CO;2-N.

12. Moita GF, Crisfield MA. A finite element formulation for 3-D continua using the co-rotational technique. International Journal for Numerical Methods in Engineering 1996; 39(22):3775-3792. DOI: 10.1002/(SICI)10970207(19961130)39:22<3775::AID-NME23>3.0.CO;2-W.

13. Rankin CC. Application of linear finite elements to finite strain using corotation. AIAA paper No AIAA-2006-1751, 47th AIAA/ASME/ASCE/ASC Structures, Structural Dynamics, and Materials Conference, Newport, Rhode Island, 2006; 1-28.

14. Areias P, Garção J, Pires EB, Infante Barbosa J. Exact corotational shell for finite strains and fracture. Computational Mechanics 2011; 48(4):385-406. DOI: 10.1007/s00466-011-0588-3.

15. Li ZX, Vu-Quoc L. An efficient co-rotational formulation for curved triangular shell element. International Journal for Numerical Methods in Engineering 2007; 72(9):1029-1062. DOI: 10.1002/nme.2064.

16. Zhou YX, Sze KY. A geometric nonlinear rotation-free triangle and its application to drape simulation. International Journal for Numerical Methods in Engineering 2012; 89(4):509-536. DOI: 10.1002/nme.3250.

17. Levy R, Gal E. The geometric stiffness of thick shell triangular finite elements for large rotations. International Journal for Numerical Methods in Engineering 2006; 65(9):1378-1402. DOI: 10.1002/nme.1491.

18. Khosravi P, Ganesan R, Sedaghati R. An efficient facet shell element for corotational nonlinear analysis of thin and moderately thick laminated composite structures. Computers and Structures 2008; 86(9):850-858. DOI: 10.1016/j.compstruc.2007.04.010.

19. Gal E, Levy R. Geometrically nonlinear analysis of shell structures using a flat triangular shell finite element. Archives of Computational Methods in Engineering 2006; 13(3):331-388. DOI: 10.1007/BF02736397.

20. Fraeijs De Veubeke BM. The dynamics of flexible bodies. International Journal of Engineering Science 1976; 14(10):895-913. DOI: 10.1016/0020-7225(76)90102-6. 
21. Felippa CA. A systematic approach to the element-independent corotational dynamics of finite elements. Report CUCAS-00-03, Center for Aerospace Structures, College of Engineering and Applied Sciences, University of Colorado at Boulder, Campus Box 429, Boulder, Colorado 80309, January 2000.

22. Jetteur P, Cescotto S. A mixed finite element for the analysis of large inelastic strains. International Journal for Numerical Methods in Engineering 1991; 31(2):229-239. DOI: 10.1002/nme.1620310203.

23. Crisfield MA. Non-linear Finite Element Analysis of Solids and Structures, Vol. 2: Advanced Topics. John Wiley \& Sons Ltd: Chichester, 1997.

24. Pajot JM, Maute K. Analytical sensitivity analysis of geometrically nonlinear structures based on the corotational finite element method. Finite Elements in Analysis and Design 2006; 42(10):900-913. DOI: 10.1016/ j.finel.2006.01.007.

25. Sze KY, Liu XH, Lo SH. Popular benchmark problems for geometric nonlinear analysis of shells. Finite Elements in Analysis and Design 2004; 40(11):1551-1569. DOI: 10.1016/j.finel.2003.11.001.

26. Frish-Fay R. Flexible Bars. Butterworths: London, 1962.

27. Batoz JL, Bathe KJ, Ho LW. A study of three-node triangular plate bending elements. International Journal for Numerical Methods in Engineering 1980; 15(12):1771-1812. DOI: 10.1002/nme.1620151205.

28. Jeyachandrabose C, Kirkhope J, Ramesh Babu C. An alternate explicit formulation for the DKT plate bending element. International Journal for Numerical Methods in Engineering 1985; 21(7):1289-1293. DOI: 10.1002/ nme.1620210709.

29. Felippa CA. A study of optimal membrane triangles with drilling freedoms. Computer Methods in Applied Mechanics and Engineering 2003; 192(16-18):2125-2168. DOI: 10.1016/S0045-7825(03)00253-6.

30. Battini JM, Pacoste C. On the choice of the linear element for corotational triangular shells. Computer Methods in Applied Mechanics and Engineering 2006; 195(44-47):6362-6377. DOI: 10.1016/j.cma.2006.01.007.

31. Battini JM. A modified corotational framework for triangular shell elements. Computer Methods in Applied Mechanics and Engineering 2007; 196(13-16):1905-1914. DOI: 10.1016/j.cma.2006.10.006.

32. Spurrier RA. Comment on "Singularity-free extraction of a quaternion from a direction-cosine matrix". Journal of Spacecraft and Rockets 1978; 15(4):255-255. DOI: 10.2514/3.57311.

33. Martins LC, Podio-Guidugli P. A variational approach to the polar decomposition theorem. Atti della Accademia Nazionale dei Lincei Rendiconti-Classe di Scienze Fisiche-Matematiche \& Naturali (8) 1979; 66(6):487-493.

34. Eriksson A, Pacoste C. Element formulation and numerical techniques for stability problems in shells. Computer Methods in Applied Mechanics and Engineering 2002; 191(35):3775-3810. DOI: 10.1016/S0045-7825(02)00288-8.

35. Chia CY. Nonlinear Analysis of Plates. McGraw-Hill International Book Co.: New York, 1980.

36. Pacoste C. Co-rotational flat facet triangular elements for shell instability analysis. Computer Methods in Applied Mechanics and Engineering 1998; 156(1-4):75-110. DOI: 10.1016/S0045-7825(98)80004-2. 